\title{
Non-existence of homogeneous Einstein metrics
}

\author{
Christoph Böhm
}

\begin{abstract}
We show that there exist infinitely many simply connected compact prime homogeneous spaces $G / H$ with infinite second homotopy group which do not admit $G$-invariant Einstein metrics.
\end{abstract}

Mathematics Subject Classification (2000). 53C25, 53C30.

Keywords. Einstein metric, homogeneous space.

A Riemannian metric $g$ on a closed manifold is called Einstein if it satisfies Einstein's equation $\operatorname{ric}_{g}=\lambda \cdot g$. Even though there exist many interesting classes of Einstein metrics, e.g. Kähler-Einstein metrics [Yau], [Tia], metrics with small holonomy group [Jo], Sasakian-Einstein metrics [BoGa] and homogeneous Einstein metrics [Heb], [BWZ], general existence and non-existence results are hard to obtain (for many more details and references see, e.g. [Bes], [LW]). For instance, in dimensions greater or equal than five no obstructions to the existence of Einstein metrics are known (cf. [LeB] for the four-dimensional case).

In this paper we examine the Einstein equation for $G$-invariant metrics on compact homogeneous spaces $G / H$. On such spaces the Einstein constant $\lambda$ of a $G$-invariant Einstein metric is non-negative (Bochner's theorem [Bo]) and zero if and only if the metric is flat [AlKi]. If the Einstein constant is positive, then the fundamental group of $G / H$ is finite by the theorem of Bonnet-Myers.

In what follows let us assume that $G / H$ is a simply connected homogeneous spaces with $G$ connected simply connected and semisimple. The homogeneous space $G / H$ is called a prime homogeneous space, if the normalizer $N_{G}(H)$ of $H$ in $G$ and $H$ have the same rank and if $G / H$ is not a product of homogeneous spaces. An arbitrary simply connected homogeneous space is either a product of prime homogeneous spaces or the total space of a principal torus bundle over such a product. In both cases, the factors of this product are called the prime factors of $G / H$.

Theorem ([Bö2]). Let $G / H$ be a compact simply connected homogeneous space with $G$ connected simply connected and semisimple. If there exists a field $\mathbb{F}$ such that the reduced homology with coefficients in $\mathbb{F}$ of the simplicial complexes of all prime factors of $G / H$ does not vanish, then $G / H$ admits a $G$-invariant Einstein metric. 
The simplicial complex of a compact homogeneous space $G / H$ is defined by certain subgroups $K$ with $H \subsetneq K \subsetneq G$ (cf. [Bö2]). This theorem shows that purely Lie-theoretical properties of the prime factors of a compact homogeneous space guarantee the existence of a homogeneous Einstein metric. Conversely, we have the following result:

Theorem A. There exist infinitely many simply connected prime homogeneous spaces $G_{p} / H_{p}$ with infinite second homotopy group, such that compact simply connected homogeneous spaces $G / H, G$ connected simply connected and semisimple, do not admit $G$-invariant Einstein metrics, if $G_{p} / H_{p}$ is a prime factor of $G / H$ and if $G / H$ is $G_{p} / H_{p}$-generic.

A homogeneous space $G / H$ is called $G_{p} / H_{p}$-generic for a prime factor $G_{p} / H_{p}$ of $G / H$, if the irreducible summands of the isotropy representation of $H_{p}$ are acted on irreducibly by $H$. Obviously, this condition is satisfied for the homogeneous space $G / H=G_{p} / H_{p}$; but, as we will see below, in general this assumption is necessary. Notice furthermore that it follows from the long homotopy sequence of the fibration $H_{p} \rightarrow G_{p} \rightarrow G_{p} / H_{p}$ that prime homogeneous spaces $G_{p} / H_{p}$ have finite second homotopy group if and only if the isotropy group $H_{p}$ is semisimple.

The spaces $G_{p} / H_{p}=\operatorname{Spin}(n) \times \operatorname{Spin}(n) / \Delta \operatorname{Spin}(n-2) \cdot(\operatorname{Spin}(2) \times \operatorname{Spin}(2))$, $n>8$, provide concrete examples for prime homogeneous spaces Theorem A can be applied to (where $\operatorname{Spin}(n)$ denotes the double cover of $\mathrm{SO}(n)$ ). The simplest examples of homogeneous spaces with such a prime factor are given by $G / H=$ $\operatorname{Spin}(n) \times \operatorname{Spin}(n) / \Delta \operatorname{Spin}(n-2) \cdot \Delta_{k, q} \operatorname{Spin}(2)$, where $k, q$ are coprime integers and $\Delta_{k, q} \operatorname{Spin}(2)$ is embedded in $\operatorname{Spin}(2) \times \operatorname{Spin}(2)$ with slope determined by $(k, q)$. The $S^{1}$-bundle $G / H$ over $G_{p} / H_{p}$ is $G_{p} / H_{p}$-generic if $(k, q) \neq \pm(1,1),(0, \pm 1),( \pm 1,0)$, and consequently such $S^{1}$-bundles do not admit $G$-invariant Einstein metrics by Theorem A.

For $(k, p)= \pm(1,1)$ the homogeneous space $G / H$ does admit a $G$-invariant Einstein metric by the Graph Theorem [BWZ]. This shows the existence of singular torus bundles $G / H$ over prime homogeneous spaces, which carry $G$-invariant Einstein metrics, even when generic torus bundles do not. The reason for this is that for singular torus bundles the dimension of the space $\mathcal{M}^{G}$ of $G$-invariant metrics on $G / H$ is strictly larger than that for generic torus bundles.

From Theorem A we deduce also the following

Corollary. For any $m \in \mathbb{N}$ there exists a simply connected compact non-product homogeneous space $G / H$ with $\operatorname{dim} \mathcal{M}^{G} \geq m$, which does not admit $G$-invariant Einstein metrics.

For all previously known non-product homogeneous spaces $G / H$ not admitting $G$-invariant Einstein metrics we have $\operatorname{dim} \mathcal{M}^{G} \leq 4$ [WZ2], [Wa], [PaSa], [BK], [DiKe]. 
To give the reader a feeling for the Einstein equation for homogeneous metrics, let us consider compact homogeneous spaces $G / H$ whose isotropy representation $\mathfrak{m}$ can be decomposed into pairwise inequivalent irreducible summands $\mathfrak{m}_{i}, 1 \leq$ $i \leq \ell$. In this special case, any $G$-invariant metric is given by $\left.\sum_{i=1}^{\ell} x_{i} Q\right|_{\mathfrak{m}_{i}}$ where $x_{1}, \ldots, x_{\ell}>0$ and $Q$ denotes a fixed bi-invariant background metric. The metric is then Einstein with Einstein constant $\lambda$ if and only if

$$
\frac{b_{i}}{2 x_{i}}-\frac{1}{2 d_{i}} \sum_{j, k=1}^{\ell}[i j k] \frac{x_{k}}{x_{i} x_{j}}+\frac{1}{4 d_{i}} \sum_{j, k=1}^{\ell}[i j k] \frac{x_{i}}{x_{j} x_{k}}=\lambda, \quad 1 \leq i \leq \ell
$$

where $b_{i} \geq 0$ and [ijk] $\geq 0$ are structure constants of $G / H$ and $d_{i}=\operatorname{dim} \mathfrak{m}_{i}$ [WZ2], [PaSa]. In order to show non-existence of homogeneous Einstein metrics one has to prove that these algebraic equations have no positive real solutions. Let us mention that no homogeneous space is known where these equations do not admit complex solutions.

Next, we describe a conceptual approach to the non-existence problem of homogeneous Einstein metrics. For a compact homogeneous space $G / H$ let $\mathfrak{p}_{1}, \ldots, \mathfrak{p}_{\ell_{*}}$ denote the isotypical summands of the isotropy representation $\mathfrak{m}=\mathfrak{p}_{1} \oplus \cdots \oplus \mathfrak{p}_{\ell_{*}}$ of the isotropy group $H$. Each isotypical summand sums up the irreducible summands of $\mathfrak{m}$ which are equivalent. By Schur's Lemma, the traceless Ricci tensor of a $G$-invariant metric on $G / H$ respects this splitting. This tensor is precisely the negative gradient vector of the Hilbert action [Hi] with respect to the natural $L^{2}$ metric. Since on closed manifolds the Hilbert action characterizes Einstein metrics variationally, a compact homogeneous space $G / H$ cannot carry $G$-invariant Einstein metrics, if the restriction of the traceless Ricci tensor to an isotypical summand is negative (positive) definite for all $G$-invariant metrics.

The next two theorems, Theorem B and Theorem C, provide Lie-theoretical properties of such homogeneous spaces:

Theorem B. Let $G / H$ be a compact homogeneous space with finite fundamental group. If for all $G$-invariant metrics on $G / H$ the restriction of the traceless Ricci tensor to an isotypical summand of the isotropy representation of $H$ is negative definite, then there exists a compact intermediate Lie group $K$ such that $G / K$ is isotropy irreducible, $\operatorname{dim} G / K>1$ and $K / H$ is a virtual product of isotropy irreducible spaces.

A compact homogeneous space $G / K$ is called isotropy irreducible, if the isotropy representation of $K$ is irreducible. We say that a homogeneous space $K / H$ splits virtually, if this is true on Lie algebra level, that is if $T_{1} K=\mathfrak{k}=\mathfrak{k}_{1} \oplus \mathfrak{k}_{2}$ and $T_{1} H=\mathfrak{h}=\mathfrak{h}_{1} \oplus \mathfrak{h}_{2}$ with $\mathfrak{h}_{i}<\mathfrak{k}_{i}$. From the classification of isotropy irreducible spaces [Wo], [WZ3] it follows that homogeneous spaces obeying the obstruction in Theorem B are very special. For instance, if both $G$ and $H$ are connected, then we 
have $\operatorname{dim} \mathcal{M}^{G} \leq 4$. Notice that the isotypical summand mentioned in Theorem B is the orthogonal complement of $\mathfrak{k}$ in $\mathfrak{g}=T_{1} G$.

Let us also mention that the homogeneous spaces described in Theorem B have been used to construct simply connected cohomogeneity one manifolds, which do not admit cohomogeneity one Einstein metrics [Bö1] but Riemannian metrics of positive Ricci curvature [GrZi].

The space $G / H=\mathrm{SU}(m+k) / \mathrm{S}(\mathrm{SO}(m) \mathrm{U}(1) \mathrm{SO}(k) \mathrm{U}(1)), m+k>4$ and $m, k \geq 2$, is a concrete example for Theorem B due to M. Wang. Non-existence for $k>m^{2}+2$ has been established in [WZ2]. In this case we have $K=\mathrm{S}(\mathrm{U}(m) \mathrm{U}(k))$, hence $K / H$ is the product of two isotropy irreducible spaces.

Theorem C. Let $G / H$ be a compact homogeneous space with finite fundamental group. If for all $G$-invariant metrics on $G / H$ the restriction of the traceless Ricci tensor to an isotypical summand of the isotropy representation is positive definite, then there exists a compact intermediate Lie group $K$ such that $K / H$ is isotropy irreducible, $\operatorname{dim} K / H>1$ and all $G$-invariant metrics on $G / H$ are submersion metrics.

A $G$-invariant metric on a homogeneous space $G / H$ is a submersion metric with respect to a submersion $\pi: G / H \rightarrow G / K ; g H \mapsto g K$ if it is given by a $K$-invariant metric on the fibre $K / H$ and a $G$-invariant metric on the base $G / K$. Since in the above situation $K / H$ is isotropy irreducible, the $K$-invariant metric on $K / H$ is uniquely determined up to scaling. Notice that the isotypical summand mentioned in Theorem $\mathrm{C}$ is the orthogonal complement of $\mathfrak{h}$ in $\mathfrak{k}$.

The space $G / H=\operatorname{Spin}(n) \times \operatorname{Spin}(n) / \Delta \operatorname{Spin}(n-k) \cdot(\operatorname{Spin}(k) \times \operatorname{Spin}(k))$, $n>k^{2}+k+2$ and $k \geq 2$, is a concrete example for Theorem C. In this case we have $K=(\operatorname{Spin}(n-k) \operatorname{Spin}(k)) \times(\operatorname{Spin}(n-k) \operatorname{Spin}(k))$. For $n=k^{2}+k+2$ there exists precisely one $G$-invariant Einstein metric, whereas for $n<k^{2}+k+2$ we have at least two non-isometric $G$-invariant Einstein metrics.

The non-existence criterion described in Theorem $\mathrm{C}$ can be generalized as follows: For a subset $I_{*}$ of $\left\{1,2, \ldots, \ell_{*}\right\}$ we consider the restriction of the Ricci tensor to the subspace $\oplus_{i \in I_{*}} \mathfrak{p}_{i}$ of $\mathfrak{m}$ and the tracefree part of this symmetric bilinear form. If the restriction of the latter bilinear form to an isotypical summand $\mathfrak{p}_{i_{0}}, i_{0} \in I_{*}$, is positive definite for all $G$-invariant metrics on $G / H$, then $G / H$ does not admit $G$-invariant Einstein metrics. The following examples indicates already that these more general obstructions cover many further homogeneous spaces:

Example. Let $G / H=\mathrm{SU}\left(m+n_{1}+\cdots+n_{k}\right) / \mathrm{S}\left(\mathrm{SO}(m) \mathrm{U}(1) \times \mathrm{U}\left(n_{1}\right) \times \cdots \times \mathrm{U}\left(n_{k}\right)\right)$, where $m, n_{1}, \ldots, n_{k} \geq 1$. If $m>\left(\sum_{i=1}^{k} n_{i}\right)^{2}+2$, then $G / H$ does not admit $G$ invariant Einstein metrics.

Note that $\operatorname{dim} \mathcal{M}^{G}=\frac{1}{2} k(k+1)+1$, that $G$ is simple and that the subgroup $K$ mentioned in Theorem C equals $\mathrm{S}\left(\mathrm{U}(m) \times \mathrm{U}\left(n_{1}\right) \times \cdots \times \mathrm{U}\left(n_{k}\right)\right)$. Whenever $n_{i}=1$, 
for at least one $i$, we obtain new examples of prime homogeneous spaces for which Theorem $\mathrm{A}$ is true.

The above obstructions turn out to be extremely flexible. They allow us to prove glueing theorems for prime homogeneous spaces Theorem A can be applied to. Suppose that $G / H$ does not admit $G$-invariant Einstein metrics by means of one of these obstructions. Pick any homogeneous space $\tilde{G} / \tilde{H}$, such that there exists a simple (or abelian) Lie group $L$ with $H=H^{\prime} L$ and $\tilde{H}=L \tilde{H}^{\prime}$. Then, under certain purely Lie-theoretical assumption on $G / H$ (made precise in Theorem 4.7) the compact homogeneous space $\hat{G} / \hat{H}=G \times \tilde{G} /\left(H^{\prime} \cdot \Delta L \cdot \tilde{H}^{\prime}\right)$ does not admit $\hat{G}$-invariant Einstein metrics. For instance we have:

Proposition. Let $G / H=\mathrm{SU}\left(m+n_{1}+\cdots+n_{k}\right) / \mathrm{S}\left(\mathrm{SO}(m) \mathrm{U}(1) \times \mathrm{U}\left(n_{1}\right) \times \cdots \times\right.$ $\left.\mathrm{U}(1) \mathrm{SU}\left(n_{k}\right)\right)$, where $m, n_{1}, \ldots, n_{k} \geq 1, n_{1}=1, n_{k} \geq 2, m>\left(\sum_{i=1}^{k} n_{i}\right)^{2}+2$. Furthermore let $\tilde{G} /\left(\mathrm{SU}\left(n_{k}\right) \tilde{H}^{\prime}\right)$ be a prime homogeneous space. Then Theorem $A$ holds true for the prime homogeneous space $G_{p} / H_{p}=G \times \tilde{G} /(\mathrm{S}(\mathrm{SO}(m) \mathrm{U}(1) \times$ $\left.\left.\mathrm{U}\left(n_{1}\right) \times \cdots \times \mathrm{U}(1)\right) \cdot \Delta \mathrm{SU}\left(n_{k}\right) \cdot \tilde{H}^{\prime}\right)$.

Finally, we explain how the previously known non-existence examples [WZ2], [Wa], [PaSa], [BK], [DiKe] fit into the above framework. Non-existence of homogeneous Einstein metrics has been described for the first time in [WZ2]. For most of these examples the isotropy representation can be decomposed into two irreducible isotypical summands; if in addition $G$ is simple such spaces have been classified recently [DiKe]. Under this assumption, the Einstein equation can be solved explicitly and the non-existence criteria given in Theorem $\mathrm{B}$ and Theorem $\mathrm{C}$ are equivalent and also necessary.

In [WZ2] also compact homogeneous spaces have been examined whose isotropy representation can be decomposed into three irreducible isotypical summands. The subgroup structure of these spaces is as described in Theorem B. However, the non-existence criterion in [WZ2] is not that given in Theorem B but one of the above mentioned generalizations. By means of Theorem $\mathrm{C}$ the homogeneous spaces $G / H=E_{7} \times E_{7} / \operatorname{Sp}(1) \Delta \operatorname{Spin}(12) \operatorname{Sp}(1)$ and $G / H=E_{8} \times E_{8} / \operatorname{Sp}(1) \Delta E_{7} \operatorname{Sp}(1)$ do not admit $G$-invariant Einstein metrics [Wa]. For the remaining two known nonexistence examples $[\mathrm{PaSa}],[\mathrm{BK}]$ non-existence does not follow from the above described obstructions.

Our paper contains 5 sections. In Section 1 we describe obstructions to the existence of homogeneous Einstein metrics. In Section 2 curvature computations are carried out. In Section 3, resp. Section 4, we prove Theorem B, resp. Theorem C. In Section 5 we present new examples of homogeneous spaces which do not admit homogeneous Einstein metrics, and we give the proof of Theorem A 


\section{The Ricci tensor of a homogeneous metric}

Let $G / H$ be a connected compact homogeneous space such that $G$ and $H$ are compact Lie groups not necessarily connected. Let $Q$ denote an $\operatorname{Ad}(G)$-invariant scalar product on $\mathfrak{g}$. Choose $\mathfrak{m}$ the $Q$-orthogonal complement to $\mathfrak{h}$ in $\mathfrak{g}$. As is well-known, every $G$-invariant metric on $G / H$ is uniquely determined by an $\operatorname{Ad}(H)$-invariant scalar product on $\mathfrak{m}$. Furthermore, for any $G$-invariant metric $g$ on $G / H$ there exists a decomposition

$$
f=\mathfrak{m}_{1} \oplus \cdots \oplus \mathfrak{m}_{\ell}
$$

of $\mathfrak{m}$ into $\operatorname{Ad}(H)$-irreducible summands, such that $g$ is diagonal with respect to $Q$, that is

$$
g=\left.\left.x_{1} Q\right|_{\mathfrak{m}_{1}} \perp \cdots \perp x_{\ell} Q\right|_{\mathfrak{m}_{\ell}}
$$

with $x_{1}, \ldots, x_{\ell}>0$. Even though the decomposition $f=\mathfrak{m}_{1} \oplus \cdots \oplus \mathfrak{m}_{\ell}$ of $\mathfrak{m}$ is not determined uniquely in general, this is true for the decomposition $\mathfrak{m}=\mathfrak{p}_{1} \oplus \cdots \oplus \mathfrak{p}_{\ell_{*}}$ of $\mathfrak{m}$ into isotypical summands. Moreover, by Schur's Lemma each $G$-invariant metric $g$ and also its Ricci tensor ric $_{g}$ respect this splitting.

Next, let us define the $\operatorname{Ad}(H)$-equivariant, $g$-selfadjoint endomorphism $\operatorname{Ric}_{g}$ by

$$
\operatorname{ric}_{g}(\cdot, \cdot)=g\left(\operatorname{Ric}_{g} \cdot, \cdot\right) .
$$

Let $I_{*}$ denote any non-empty subset of $\left\{1,2, \ldots, \ell_{*}\right\}$ and let $\mathfrak{p}_{I_{*}}=\oplus_{i \in I_{*}} \mathfrak{p}_{i}$. We consider the restriction $\left(\operatorname{Ric}_{g}\right)_{I_{*}}$ of $\operatorname{Ric}_{g}$ to $\mathfrak{p}_{I_{*}}$ as an endomorphism of $\mathfrak{p}_{I_{*}}$. Let $\operatorname{sc}(g)_{I_{*}}=\operatorname{tr}\left(\operatorname{Ric}_{g}\right)_{I_{*}}$ and let

$$
\left(\left(\operatorname{Ric}_{g}\right)_{I_{*}}\right)^{0}=\left(\operatorname{Ric}_{g}\right)_{I_{*}}-\frac{\operatorname{sc}(g)_{I_{*}}}{\operatorname{dim} \mathfrak{p}_{I_{*}}} \cdot \operatorname{id}_{\mathfrak{p}_{I_{*}}}
$$

denote the tracefree part of $\left(\operatorname{Ric}_{g}\right)_{I_{*}}$. We call $\left(\left(\left(\operatorname{Ric}_{g}\right)_{I_{*}}\right)^{0}\right)_{i_{0}}$ negative (positive) definite, if the symmetric 2-form $g\left(\left(\left(\left(\operatorname{Ric}_{g}\right)_{I_{*}}\right)^{0}\right)_{i_{0}} \cdot, \cdot\right)$ on $\mathfrak{p}_{i_{0}}$ is negative (positive) definite.

Since $G$-invariant Einstein metrics on $G / H$ are characterized variationally as the critical points of the Hilbert action restricted to the space of $G$-invariant metrics of volume 1 (cf. [Bes]), we obtain the following obstructions to existence of $G$-invariant Einstein metrics.

Lemma 1.2. Let $G / H$ be a compact homogeneous space. Let $I_{*} \subset\left\{1,2, \ldots, \ell_{*}\right\}$, $\left|I_{*}\right| \geq 2$ and $i_{0} \in I_{*}$. If for all $G$-invariant metrics $g$ on $G / H$ the endomorphism $\left(\left(\left(\operatorname{Ric}_{g}\right)_{I_{*}}\right)^{0}\right)_{i_{0}}: \mathfrak{p}_{i_{0}} \rightarrow \mathfrak{p}_{i_{0}}$ is negative (positive) definite, then $G / H$ does not admit $G$-invariant Einstein metrics. 
Next, we present a well-known formula for the Ricci tensor of a homogeneous metric on a compact homogeneous space. Let $g \in \mathcal{N}^{G}$ and let $f=\mathfrak{m}_{1} \oplus \cdots \oplus \mathfrak{m}_{\ell}$ be a decomposition of $\mathfrak{m}$, which diagonalizes $g$. Then by [WZ2], [PaSa] we have

$$
\begin{aligned}
& \left(\operatorname{Ric}_{g}\right)_{m m} \\
& =\left(\frac{1}{2} \frac{b_{m}}{x_{m}}-\frac{1}{2 d_{m}} \sum_{j, k=1}^{\ell}[j k m]_{f} \frac{x_{k}}{x_{m} x_{j}}+\frac{1}{4 d_{m}} \sum_{j, k=1}^{\ell}[j k m]_{f} \frac{x_{m}}{x_{j} x_{k}}\right) \cdot \mathrm{id}_{\mathfrak{m}_{m}},
\end{aligned}
$$

where $\left(\operatorname{Ric}_{g}\right)_{m m}$ denotes the restriction of $\operatorname{Ric}_{g}$ to $\mathfrak{m}_{m}$, i.e. $g\left(\left(\operatorname{Ric}_{g}\right)_{m m} X, X\right)=$ $\operatorname{ric}_{g}(X, X)$ for all $X \in \mathfrak{m}_{m}$. Here,

$$
-\left.B\right|_{\mathfrak{m}_{m}}=\left.b_{m} Q\right|_{\mathfrak{m}_{m}} \quad \text { and } \quad d_{m}=\operatorname{dim} \mathfrak{m}_{m},
$$

where $B$ denotes the Killing form on $\mathfrak{g}$. The structure constants $[i j k]_{f}$ with respect to the decomposition $f$ are defined as follows:

$$
[i j k]_{f}=\sum Q\left(\left[\hat{e}_{\alpha}, \hat{e}_{\beta}\right], \hat{e}_{\gamma}\right)^{2}
$$

where the sum is taken over $\left\{\hat{e}_{\alpha}\right\},\left\{\hat{e}_{\beta}\right\}$, and $\left\{\hat{e}_{\gamma}\right\}, Q$-orthonormal bases for $\mathfrak{m}_{i}, \mathfrak{m}_{j}$ and $\mathfrak{m}_{k}$, respectively. Notice that $[i j k]_{f}$ is invariant under permutation of $i, j, k$.

The only known relations among these structure constants have been described in [WZ2]:

$$
d_{i} b_{i}=2 d_{i} c_{i}+\sum_{j, k=1}^{\ell}[i j k]_{f}, \quad 1 \leq i \leq \ell .
$$

The nonnegative constants $c_{i}$ are given by $C_{\mathfrak{m}_{i},\left.Q\right|_{\mathfrak{h}}}=c_{i} \cdot \mathrm{id}_{\mathfrak{m}_{i}}$ where

$$
C_{\mathfrak{m}_{i},\left.Q\right|_{\mathfrak{h}}}=-\sum_{i} \operatorname{ad} z_{i} \circ \text { ad } z_{i},
$$

$\left\{z_{i}\right\} Q$-orthonormal basis of $\mathfrak{h}$, denotes the Casimir operator on $\mathfrak{m}_{i}$.

\section{The tracefree part of the Ricci tensor}

In this section we will compute the diagonal part of the tracefree part of the Ricci tensor of a homogeneous metric $g$ on a compact homogeneous space $G / H$ and more general the diagonal part of the endomorphisms $\left(\left(\left(\operatorname{Ric}_{g}\right)_{I_{*}}\right)^{0}\right)_{i_{0}}: \mathfrak{p}_{i_{0}} \rightarrow \mathfrak{p}_{i_{0}}$.

Let $g \in \mathcal{M}^{G}$ be a $G$-invariant metric on $G / H$ and let $f=\mathfrak{m}_{1} \oplus \cdots \oplus \mathfrak{m}_{\ell}$ be a decomposition of $\mathfrak{m}$, which diagonalizes $g$. For $I_{*} \subset\left\{1,2, \ldots, \ell_{*}\right\}$ let $I$ be the subset of $\{1,2, \ldots, \ell\}$ with $\mathfrak{m}_{I}=\oplus_{i \in I} \mathfrak{m}_{i}=\mathfrak{p}_{I_{*}}$. 
In order to keep notation as simple as possible let us introduce the following notations: We will write $[i j k]$ instead of $[i j k]_{f}$ and $\sum_{j, k}$ instead of $\sum_{j, k=1}^{\ell}$. If we write $\sum_{i, j, k \neq m}$, then we are summing over all indices $i, j, k$ from 1 to $\ell$ with $i, j, k \neq m$ but the last one. Thus $m$ is always fixed. If we write $\sum_{i \neq j \neq k}$, then we are summing over all indices $i, j, k$ which are pairwise distinct.

Since by (1.3)

$$
\operatorname{sc}(g)_{I_{*}}=\frac{1}{2} \sum_{i \in I} \frac{d_{i} b_{i}}{x_{i}}-\frac{1}{2} \sum_{i \in I, j, k}[i j k] \frac{x_{k}}{x_{i} x_{j}}+\frac{1}{4} \sum_{i \in I, j, k}[i j k] \frac{x_{i}}{x_{j} x_{k}},
$$

we obtain for $m \in I$

$$
\begin{aligned}
& \left(\left(\left(2 \mathrm{Ric}_{g}\right)_{I_{*}}\right)^{0}\right)_{m m} \\
& =\left(\frac{b_{m}}{x_{m}}\left(1-\frac{d_{m}}{d_{I}}\right)-\frac{1}{d_{I}} \sum_{i \in I \neq m} \frac{d_{i} b_{i}}{x_{i}}-\frac{1}{d_{m}} \sum_{i, j}[i j m] \frac{x_{j}}{x_{i} x_{m}}\right. \\
& \left.\quad+\frac{1}{2 d_{m}} \sum_{i, j}[i j m] \frac{x_{m}}{x_{i} x_{j}}+\frac{1}{d_{I}} \sum_{i \in I, j, k}[i j k] \frac{x_{k}}{x_{j} x_{i}}-\frac{1}{2 d_{I}} \sum_{i \in I, j, k}[i j k] \frac{x_{i}}{x_{j} x_{k}}\right) \cdot \operatorname{id}_{\mathfrak{m}_{m}}
\end{aligned}
$$

where $d_{I}=\sum_{i \in I} d_{i}$. We are going to extract $x_{m}$ in this formula. The third and the fourth term of (2.1) can be decomposed as follows:

$$
\begin{aligned}
& \sum_{i, j}[i j m] \frac{x_{j}}{x_{i} x_{m}} \\
& =\frac{1}{x_{m}} \sum_{i}[i i m]+\sum_{i \neq m}[i m m] \frac{1}{x_{i}}+\frac{1}{x_{m}^{2}} \sum_{i \neq m}[i m m] x_{i}+\frac{1}{x_{m}} \sum_{i \neq j \neq m}[i j m] \frac{x_{j}}{x_{i}} \\
& \sum_{i, j}[i j m] \frac{x_{m}}{x_{i} x_{j}} \\
& =\frac{1}{x_{m}}[m m m]+x_{m} \sum_{i \neq m}[i i m] \frac{1}{x_{i}^{2}}+2 \sum_{i \neq m}[i m m] \frac{1}{x_{i}}+x_{m} \sum_{i \neq j \neq m}[i j m] \frac{1}{x_{i} x_{j}} .
\end{aligned}
$$

In order to treat the fifth term in (2.1) we observe:

$$
\begin{gathered}
\sum_{i \in I, j, k}[i j k] \frac{x_{k}}{x_{i} x_{j}}=\frac{1}{x_{m}}[m m m]+\sum_{i \in I \neq m}[i i i] \frac{1}{x_{i}}+\frac{1}{x_{m}} \sum_{k \neq m}[k k m]+\sum_{i \in I \neq m, k \neq i}[i k k] \frac{1}{x_{i}} \\
+\frac{1}{x_{m}} \sum_{i \in I \neq m}[i i m]+\sum_{j \neq m, i \in I \neq j}[i i j] \frac{1}{x_{j}}+\frac{1}{x_{m}^{2}} \sum_{k \neq m}[k m m] x_{k}
\end{gathered}
$$


Vol. 80 (2005)

$$
\begin{aligned}
& +x_{m} \sum_{i \in I \neq m}[i i m] \frac{1}{x_{i}^{2}}+\sum_{i \in I \neq k \neq m}[i i k] \frac{x_{k}}{x_{i}^{2}}+\frac{1}{x_{m}} \sum_{j \neq k \neq m}[j k m] \frac{x_{k}}{x_{j}} \\
& +\frac{1}{x_{m}} \sum_{i \in I \neq k \neq m}[i k m] \frac{x_{k}}{x_{i}}+x_{m} \sum_{i \in I \neq j \neq m}[i j m] \frac{1}{x_{i} x_{j}}+\sum_{i \in I \neq j \neq k \neq m}[i j k] \frac{x_{k}}{x_{i} x_{j}} .
\end{aligned}
$$

The last term in (2.1) can be written as follows:

$$
\begin{aligned}
\sum_{i \in I, j, k}[i j k] \frac{x_{i}}{x_{j} x_{k}} & \frac{1}{x_{m}}[m m m]+\sum_{i \in I \neq m}[i i i] \frac{1}{x_{i}}+\sum_{k \neq m}[k k m] \frac{x_{m}}{x_{k}^{2}}+\frac{1}{x_{m}^{2}} \sum_{i \in I \neq m}[i m m] x_{i} \\
& +\sum_{i \in I \neq k \neq m}[i k k] \frac{x_{i}}{x_{k}^{2}}+2 \sum_{i \in I \neq m} \frac{[i i m]}{x_{m}}+2 \sum_{j \neq m, i \in I \neq j} \frac{[i j]}{x_{j}} \\
& +\sum_{j \neq k \neq m}[j k m] \frac{x_{m}}{x_{j} x_{k}}+2 \sum_{i \in I \neq k \neq m}[i k m] \frac{x_{i}}{x_{m} x_{k}}+\sum_{i \in I \neq j \neq k \neq m}[i j k] \frac{x_{i}}{x_{j} x_{k}} .
\end{aligned}
$$

We obtain

$$
\begin{aligned}
\left(\left(\left(2 \mathrm{Ric}_{g}\right)_{I_{*}}\right)^{0}\right)_{m m} & =\frac{1}{d_{I}} \cdot\left(\frac{1}{x_{m}^{2}}\left\{\left(1-\frac{d_{I}}{d_{m}}\right) \sum_{i \neq m}[i m m] x_{i}-\frac{1}{2} \sum_{i \in I \neq m}[i m m] x_{i}\right\}\right. \\
& +\frac{1}{x_{m}}\left\{\left(\frac{d_{I}}{d_{m}}-1\right) d_{m} b_{m}-\frac{d_{I}}{d_{m}} \sum_{i}[i i m]+\frac{1}{2}\left(\frac{d_{I}}{d_{m}}+1\right)[\mathrm{mmm}]+\sum_{k \neq m}[k k m]\right\} \\
+ & \frac{1}{x_{m}}\left\{\left(1-\frac{d_{I}}{d_{m}}\right) \sum_{j \neq k \neq m}[j k m] \frac{x_{k}}{x_{j}}+\sum_{i \in I \neq k \neq m}[i k m]\left(\frac{x_{k}}{x_{i}}-\frac{x_{i}}{x_{k}}\right)\right\} \\
& -\sum_{i \in I \neq m} \frac{d_{i} b_{i}}{x_{i}}+\frac{1}{2} \sum_{i \in I \neq m}[i i i] \frac{1}{x_{i}}+\sum_{i \in I \neq m, k \neq i}[i k k] \frac{1}{x_{i}}+\sum_{i \in I \neq k \neq m}[i i k] \frac{x_{k}}{x_{i}^{2}} \\
+ & \sum_{i \in I \neq j \neq k \neq m}[i j k] \frac{x_{k}}{x_{i} x_{j}}-\frac{1}{2} \sum_{i \in I \neq k \neq m}[i k k] \frac{x_{i}}{x_{k}^{2}}-\frac{1}{2} \sum_{i \in I \neq j \neq k \neq m}[i j k] \frac{x_{i}}{x_{j} x_{k}} \\
+ & x_{m}\left\{\frac{1}{2}\left(\frac{d_{I}}{d_{m}}-1\right) \sum_{i \neq m}[i i m] \frac{1}{x_{i}^{2}}+\frac{1}{2}\left(\frac{d_{I}}{d_{m}}-1\right) \sum_{j \neq k \neq m}[j k m] \frac{1}{x_{j} x_{k}}\right. \\
& \left.\left.+\sum_{i \in I \neq m}[i i m] \frac{1}{x_{i}^{2}}+\sum_{i \in I \neq j \neq m}[i j m] \frac{1}{x_{i} x_{j}}\right\}\right) \cdot \mathrm{id}_{\mathfrak{m}_{m}} .
\end{aligned}
$$


In particular, this yields the following formula for the tracefree part of the Ricci tensor:

$$
\begin{aligned}
\left(\left(2 \operatorname{Ric}_{g}\right)^{0}\right)_{m m} & \frac{1}{n} \cdot\left(\frac{1}{x_{m}^{2}}\left(\frac{1}{2}-\frac{n}{d_{m}}\right) \sum_{i \neq m}[i m m] x_{i}-\sum_{i \neq m} \frac{1}{x_{i}}\left\{d_{i} b_{i}+\frac{1}{2}[i i i]-\sum_{k}[i k k]\right\}\right. \\
& +\frac{1}{x_{m}}\left(\frac{n}{d_{m}}-1\right)\left\{d_{m} b_{m}+\frac{1}{2}[m m m]-\sum_{i}[i i m]-\sum_{j \neq k \neq m}[j k m] \frac{x_{k}}{x_{j}}\right\} \\
& +\frac{1}{2} \sum_{i \neq k \neq m}[i i k] \frac{x_{k}}{x_{i}^{2}}+\frac{1}{2} \sum_{i \neq j \neq k \neq m}[i j k] \frac{x_{k}}{x_{i} x_{j}} \\
& \left.+x_{m} \frac{1}{2}\left(\frac{n}{d_{m}}+1\right) \sum_{j, k \neq m}[j k m] \frac{1}{x_{j} x_{k}}\right) \cdot \operatorname{id}_{\mathfrak{m}_{m}},
\end{aligned}
$$

where

$$
n=d_{\{1,2, \ldots, \ell\}}=\operatorname{dim} G / H .
$$

From (1.4) we deduce the following identity:

$$
d_{i} b_{i}+\frac{1}{2}[i i i]-\sum_{k}[i k k]=2 d_{i} c_{i}+\frac{1}{2}[i i i]+\sum_{j \neq k}[i j k] \geq 0 .
$$

Equality holds if and only if $\mathfrak{m}_{i}$ is almost trivial, $[i i i]=0$ and $\left[\mathfrak{m}_{i}, \mathfrak{m}_{j}\right] \subset \mathfrak{m}_{j}$ for all $j=1,2, \ldots, \ell$. We call an irreducible summand $\mathfrak{m}_{i}$ almost trivial, if $\left[\mathfrak{h}, \mathfrak{m}_{i}\right]=0$, that is if $\mathfrak{m}_{i}$ is contained in the normalizer of $\mathfrak{h}$ in $\mathfrak{g}$.

\section{The negative definite case}

In this section we will assume that $\left(\left(\left(\operatorname{Ric}_{g}\right)_{I_{*}}\right)^{0}\right)_{i_{0}}$ is negative definite for all $g \in \mathcal{M}^{G}$. As above, let $f$ be a fixed decomposition of $\mathfrak{m}$ and let the $G$-invariant metric $g$ be given as in (1.1). Under this assumption, if $\mathfrak{m}_{m} \subset \mathfrak{p}_{i_{0}}$, then $\left(\left(\left(\operatorname{Ric}_{g}\right)_{I_{*}}\right)^{0}\right)_{m m}<0$ for all $g \in \mathcal{M}^{G}$.

If we let tend $x_{m}$ to $+\infty$ while keeping $x_{i}$ fixed for $i \neq m$, then considering the last term $x_{m}\{\ldots\}$ in (2.2) yields

$$
[i j m]=0 \quad \text { for } i, j \neq m .
$$

Hence

$$
\mathfrak{l}:=\mathfrak{h} \oplus \bigoplus_{i \neq m} \mathfrak{m}_{i}
$$


is an $H$-subalgebra, that is a proper $\operatorname{Ad}(H)$-invariant subalgebra of $\mathfrak{g}$, which contains $\mathfrak{h}$ properly. Equation (2.2) simplifies to

$$
\begin{aligned}
\left(\left(\left(2 \mathrm{Ric}_{g}\right)_{I_{*}}\right)^{0}\right)_{m m} & \frac{1}{d_{I}} \cdot\left(\frac{1}{x_{m}^{2}}\left\{\left(\frac{1}{2}-\frac{d_{I}}{d_{m}}\right) \sum_{i \in I \neq m}[i m m] x_{i}+\left(1-\frac{d_{I}}{d_{m}}\right) \sum_{k \in I^{C}}[k m m] x_{k}\right\}\right. \\
& +\frac{1}{x_{m}}\left(\frac{d_{I}}{d_{m}}-1\right)\left(d_{m} b_{m}-\frac{1}{2}[m m m]\right)-\sum_{i \in I \neq m} \frac{1}{x_{i}}\left\{d_{i} b_{i}+\frac{1}{2}[i i i]-\sum_{k}[i k k]\right\} \\
& +\frac{1}{2} \sum_{i \in I \neq k \in I \neq m}[i i k] \frac{x_{k}}{x_{i}^{2}}+\sum_{i \in I \neq m, k \in I^{C}}[i i k] \frac{x_{k}}{x_{i}^{2}}-\frac{1}{2} \sum_{i \in I \neq m, k \in I^{C}}[i k k] \frac{x_{i}}{x_{k}^{2}} \\
& +\frac{1}{2} \sum_{i \in I \neq j \in I \neq k \in I \neq m}[i j k] \frac{x_{k}}{x_{i} x_{j}}+\sum_{i \in I \neq j \in I \neq m, k \in I^{C}}[i j k] \frac{x_{k}}{x_{i} x_{j}} \\
& \left.+\sum_{i \in I \neq m, j \in I^{C} \neq k \in I^{C}}[i j k] \frac{x_{k}}{x_{i} x_{j}}-\frac{1}{2} \sum_{i \in I \neq m, j \in I^{C} \neq k \in I^{C}}[i j k] \frac{x_{i}}{x_{j} x_{k}}\right) \cdot \mathrm{id}_{\mathfrak{m}_{m}}
\end{aligned}
$$

where $I^{C}=\{1, \ldots, \ell\} \backslash I$. Let $k \in I^{C}$. If we set $x_{k}=x_{m}$ and let $x_{k}$ tend to $+\infty$ while keeping $x_{i}$ constant for $i \neq k, m$, then we get

$$
\begin{aligned}
{[i j k]=0 } & \text { for } i, j \in I \backslash\{m\}, k \in I^{C} \\
{\left[i k k^{\prime}\right]=0 } & \text { for } i \in I \backslash\{m\}, k, k^{\prime} \in I^{C}, k \neq k^{\prime} .
\end{aligned}
$$

Since by (3.1) $[i j m]=0$ for $i, j \neq m,(3.3)$ implies that

$$
\mathfrak{k}=\mathfrak{h} \oplus \bigoplus_{i \in I \backslash\{m\}} \mathfrak{m}_{i}
$$

is an $H$-subalgebra. We have $\mathfrak{k} \leq \mathfrak{l}$ and $\mathfrak{k}=\mathfrak{l}$ if and only if $I_{*}=\left\{1,2, \ldots, \ell_{*}\right\}$.

Let now $w_{k}=\frac{x_{k}}{x_{m}}$ for $k \neq m$. By (3.3) and (3.4), equation (3.2) simplifies to

$$
\begin{aligned}
& \left(\left(\left(2 \operatorname{Ric}_{g}\right)_{I_{*}}\right)^{0}\right)_{m m} \cdot x_{m} \\
& =\frac{1}{d_{I}} \cdot\left(\left(\frac{1}{2}-\frac{d_{I}}{d_{m}}\right) \sum_{i \in I \neq m}[i m m] w_{i}+\left(1-\frac{d_{I}}{d_{m}}\right) \sum_{k \in I^{C}}[k m m] w_{k}\right. \\
& \quad+\left(\frac{d_{I}}{d_{m}}-1\right)\left(d_{m} b_{m}-\frac{1}{2}[\mathrm{mmm}]\right)-\sum_{i \in I \neq m} \frac{1}{w_{i}}\left\{d_{i} b_{i}+\frac{1}{2}[i i i]-\sum_{k}[i k k]\right\}
\end{aligned}
$$




$$
\begin{array}{r}
+\frac{1}{2}\left\{\sum_{i \in I \neq k \in I \neq m}[i i k] \frac{w_{k}}{w_{i}^{2}}-\sum_{i \in I \neq m, k \in I^{C}}[i k k] \frac{w_{i}}{w_{k}^{2}}\right. \\
\left.\left.+\sum_{i \in I \neq j \in I \neq k \in I \neq m}[i j k] \frac{w_{k}}{w_{i} w_{j}}\right\}\right) \cdot \mathrm{id}_{\mathfrak{m}_{m}} .
\end{array}
$$

Let $i \in I \backslash\{m\}$ and let $w_{i}$ tend to zero while keeping $w_{k}$ constant for $k \neq i$. We get

$$
[i j k]=0 \quad \text { for } i, j, k \in I \backslash\{m\}, i, j \neq k \text {. }
$$

Hence equation (3.2) simplifies further to

$$
\begin{aligned}
& \left(\left(\left(2 \operatorname{Ric}_{g}\right)_{I_{*}}\right)^{0}\right)_{m m} \cdot x_{m} \\
& =\frac{1}{d_{I}} \\
& \cdot\left(\left(\frac{1}{2}-\frac{d_{I}}{d_{m}}\right) \sum_{i \in I \neq m}[i m m] w_{i}-\sum_{i \in I \neq m} \frac{1}{w_{i}}\left(d_{i} b_{i}+\frac{1}{2}[i i i]-\sum_{k}[i k k]\right)\right. \\
& \quad+\left(\frac{d_{I}}{d_{m}}-1\right)\left\{d_{m} b_{m}-\frac{1}{2}[m m m]-\sum_{k \in I^{C}}[k m m] w_{k}\right\} \\
& \left.\quad-\frac{1}{2} \sum_{i \in I \neq m, k \in I^{C}}[i k k] \frac{w_{i}}{w_{k}^{2}}\right) \cdot \operatorname{id}_{\mathfrak{m}_{m}} .
\end{aligned}
$$

Recall that an $H$-subalgebra $\mathfrak{k}$ is called toral if $\mathfrak{k}$ is an abelian extension of $\mathfrak{h}$, otherwise non-toral. From (3.5) we deduce that there exists a unique decomposition $\mathfrak{k}=\mathfrak{z}^{\prime}(\mathfrak{k}) \oplus$ $\bigoplus_{i=1}^{r} \mathfrak{k}_{i} \oplus \mathfrak{r}$ of $\mathfrak{k}$, where $\mathfrak{k}_{i}$ are non-toral $H$-subalgebras, and a unique decomposition $\mathfrak{h}=\bigoplus_{i=1}^{r} \mathfrak{h}_{i} \oplus \mathfrak{r}$ of $\mathfrak{h}$, where $\mathfrak{h}_{i}<\mathfrak{k}_{i}$, such that the $Q$-orthogonal complement $\mathfrak{q}_{i}$ of $\mathfrak{h}_{i}$ in $\mathfrak{k}_{i}$ is an isotypical summand of the $\operatorname{Ad}(H)$-module $\mathfrak{k}=\mathfrak{z}^{\prime}(\mathfrak{k}) \oplus \bigoplus_{i=1}^{r} \mathfrak{q}_{i}$ (cf. [WZ2, Theorem 2.1]).

For an $H$-subalgebra $\mathfrak{k}$ of $\mathfrak{g}$ let now $H(\mathfrak{k})$ denote the smallest subgroup of $G$ with Lie algebra $\mathfrak{k}$ containing $H$. Then it follows that $H(\mathfrak{k}) / H$ splits virtually into a product of isotropy irreducible spaces

$$
H(\mathfrak{k}) / H=T^{k} \times \prod_{i=1}^{r} H\left(\mathfrak{k}_{i}\right) / H_{i},
$$

and we obtain the following inclusions of intermediate Lie groups:

$$
H<H(\mathfrak{k}) \leq H(\mathfrak{l})<G,
$$

where $G / H(\mathfrak{l})$ is virtually isotropy irreducible and $H(\mathfrak{k}) / H$ is a virtual product of isotropy irreducible spaces.

Next, we examine the case when $\mathfrak{p}_{i_{0}}$ is not irreducible. Suppose that there exists $\mathfrak{m}^{\prime} \in I$ with $\mathfrak{m} \neq \mathfrak{m}^{\prime}$, such that $\mathfrak{m}_{m}$ and $\mathfrak{m}_{m^{\prime}}$ are equivalent. Performing the above 
computation for $\mathfrak{m}^{\prime}$, by (3.6) we get $\left[\mathfrak{m}_{m}, \mathfrak{m}_{m}\right] \subset \mathfrak{m}_{m} \oplus \mathfrak{h}$, hence $[\mathrm{imm}]=0$ for $i \neq m$. Therefore, $G / H$ splits virtually as $G / H=\tilde{G}_{1} / \tilde{H}_{1} \times \tilde{G}_{2} / \tilde{H}_{2}$ where $\tilde{G}_{1} / \tilde{H}_{1}$ and $\tilde{G}_{2} / \tilde{H}_{2}$ correspond to $\mathfrak{m}_{m}$ and $\oplus_{i \neq m} \mathfrak{m}_{i}$, respectively. By carrying out the same computation for $\mathfrak{m}_{m^{\prime}}$ we finally obtain $\left[\mathfrak{p}_{i_{0}}, \oplus_{i=1, i \neq i_{0}}^{\ell_{*}} \mathfrak{p}_{i}\right]=0$. Therefore $G / H$ splits virtually as $G / H=G_{1} / H_{1} \times G_{2} / H_{2}$ where $G_{1} / H_{1}$ and $G_{2} / H_{2}$ correspond to $\mathfrak{p}_{i_{0}}$ and $\oplus_{i=1, i \neq i_{0}}^{\ell_{*}} \mathfrak{p}_{i}$, respectively. Note that $G_{1} / H_{1}$ splits virtually into a product of isotropy irreducible spaces. It follows that virtually $G_{1} / H_{1}=T^{k}$ for $k \geq 2$ (cf. [WZ2, Theorem 2.1]).

Proposition 3.7. Let $G / H$ be a compact homogeneous space. Let $I_{*} \subset\left\{1,2, \ldots, \ell_{*}\right\}$ and $i_{0} \in I_{*}$. Suppose that $\left(\left(\left(\operatorname{Ric}_{g}\right)_{I_{*}}\right)^{0}\right)_{i_{0}}$ is negative definite for all $G$-invariant metrics $g$ on $G / H$. If $\mathfrak{p}_{i_{0}}$ is not irreducible, then virtually $G / H=T^{k} \times G_{2} / H_{2}$ for $k \geq 2$. Furthermore, if $\mathfrak{p}_{i_{0}}$ is irreducible and $\mathfrak{h} \oplus \mathfrak{p}_{i_{0}}$ is a toral $H$-subalgebra, the same is true for $k \geq 1$.

In the remaining part of this section we will assume $\left|\pi_{1}(G / H)\right|<\infty$. In this case it follows from the above proposition that $\mathfrak{p}_{i_{0}}$ is irreducible and that $\mathfrak{h} \oplus \mathfrak{p}_{i_{0}}$ is not a toral subalgebra of $\mathfrak{g}$. Therefore, the Lie subgroup $L=H(\mathfrak{l})$ is compact with $\operatorname{dim} G / L>1$. Since in case $I_{*}=\left\{1,2, \ldots, \ell_{*}\right\}$ we have $\mathfrak{k}=\mathfrak{l}$, we obtain the proof of Theorem B.

Next, we will focus on the case $I_{*}=\left\{1,2, \ldots, \ell_{*}\right\}$. Since then $I^{C}=\emptyset$, equation (3.2) simplifies further to

$$
\begin{aligned}
& \left(\left(\left(2 \operatorname{Ric}_{g}\right)_{I_{*}}\right)^{0}\right)_{m m} \cdot x_{m} \cdot n \\
& =\left(n-d_{m}\right) \cdot\left(b_{m}-\frac{[m m m]}{2 d_{m}}\right) \\
& \quad-\sum_{i \neq m} \frac{1}{w_{i}}\left\{\left(\frac{n}{d_{m}}-\frac{1}{2}\right)[i m m] w_{i}^{2}+d_{i} b_{i}-\frac{1}{2}[i i i]-[i m m]\right\} .
\end{aligned}
$$

By the above discussion, [iii ] and [imm], $1 \leq i \leq \ell$, are the only non-zero structure constants with respect to the decomposition $f$, fixed in the very beginning.

Finally, we will investigate which of these structure constants can vanish. Since $\left|\pi_{1}(G / H)\right|<\infty$, we have $d_{m} b_{m}-\frac{1}{2}[\mathrm{mmm}]>0$ (cf. [Bö2, Corollary 4.17]). If there exists $i \neq m$ with $[i m m]=0$, then $G / H$ splits virtually. Therefore, we may assume in the following $[\mathrm{imm}]>0$ for all $i$. We have $d_{i} b_{i}-\frac{1}{2}[i i i]-[\mathrm{imm}]=0$ for $i \neq m$ if and only if $\mathfrak{h} \oplus \mathfrak{m}_{i}$ is a toral $H$-subalgebra.

Let $I_{\neq 0}$ denote the set of indices $i \in\{1,2, \ldots, \ell\} \backslash\{m\}$, such that $d_{i} b_{i}-\frac{1}{2}[i i i]-$ $[\mathrm{imm}]$ is positive. If $I_{\neq 0}=\{1,2, \ldots, \ell\} \backslash\{m\}$, then the assumption $\left(\left(\left(2 \operatorname{Ric}_{g}\right)_{I_{*}}\right)^{0}\right)_{i_{0}}$ 
being negative definite for all $g \in \mathcal{M}^{G}$ is equivalent to the following inequality:

$$
\begin{aligned}
\frac{1}{2}\left(b_{m}\right. & \left.-\frac{[m m m]}{2 d_{m}}\right) \\
& <\sum_{i \neq m} \sqrt{\left(d_{i} b_{i}-\frac{[i i i]}{2}-[i m m]\right) \cdot \frac{[i m m]}{n-d_{m}} \cdot\left(\frac{1}{2\left(n-d_{m}\right)}+\frac{1}{d_{m}}\right)}
\end{aligned}
$$

It follows as in the proof of [WZ2, Theorem 2.1], that this inequality does not depend on the choice of the decomposition $f$. If $I_{\neq 0}$ is a proper subset of $\{1,2, \ldots, \ell\} \backslash\{m\}$, then the above assumption is equivalent to the fact, that an inequality is satisfied obtained from (3.8) be replacing $<$ by $\leq$ and summing over $i \in I_{\neq 0}$. Again this inequality does not depend on the choice of the decomposition $f$.

Remark 3.9. It would be very interesting to understand inequality (3.8) from a qualitative point of view. Notice that for $\ell=m=2$ inequality (3.8) is nothing by (5.1).

\section{The positive definite case}

In this section we will assume that $\left(\left(\left(\operatorname{Ric}_{g}\right)_{I_{*}}\right)^{0}\right)_{i_{0}}$ is positive definite for all $g \in \mathcal{M}^{G}$. As above, let $f$ be a fixed decomposition of $\mathfrak{m}$ which diagonalizes $g$ (cf. (1.1)). Under this assumption, if $\mathfrak{m}_{m} \subset \mathfrak{p}_{i_{0}}$, then $\left(\left(\left(\operatorname{Ric}_{g}\right)_{I_{*}}\right)^{0}\right)_{m m}>0$ for all $g \in \mathcal{M}^{G}$.

If we let tend $x_{m}$ to 0 while keeping $x_{i}$ fixed for $i \neq m$, then considering the first term $\frac{1}{x_{m}^{2}}\{\ldots\}$ in (2.2) yields $[i m m]=0$ for $i \neq m$. Hence

$$
\mathfrak{k}=\mathfrak{h} \oplus \mathfrak{m}_{m}
$$

is an $H$-subalgebra. Moreover, we claim

$$
[j k m]=0 \quad \text { for } j \neq k .
$$

To see this, we consider the third term $\frac{1}{x_{m}}\{\ldots\}$ in (2.2). We have

$$
\begin{aligned}
\left(1-\frac{d_{I}}{d_{m}}\right) \sum_{j \neq k \neq m}[j k m] \frac{x_{k}}{x_{j}}+\sum_{i \in I \neq k \neq m}[i k m]\left(\frac{x_{k}}{x_{i}}-\frac{x_{i}}{x_{k}}\right) & -\frac{d_{I}}{d_{m}}\left\{\sum_{j \in I^{C} \neq k \in I^{C}}[j k m] \frac{x_{k}}{x_{j}}+\sum_{i \in I \neq m, k \in I^{C}}[i k m]\left(\frac{x_{k}}{x_{i}}+\frac{x_{i}}{x_{k}}\right)+\sum_{i \in I \neq j \in I \neq m}[i j m] \frac{x_{i}}{x_{j}}\right\} \\
& +\sum_{j \in I^{C} \neq k \in I^{C}}[j k m] \frac{x_{k}}{x_{j}}+2 \sum_{i \in I \neq m, k \in I^{C}}[i k m] \frac{x_{k}}{x_{i}}+\sum_{i \in I \neq j \in I \neq m}[i j m] \frac{x_{i}}{x_{j}}
\end{aligned}
$$


First of all, $[j k m]=0$ for $j, k \in I^{C}, j \neq k$, since if we let $x_{k}$ tend to $+\infty$, while keeping $x_{j}$ fixed for $j \in I^{C}, j \neq k$, and if we set $x_{i}=x_{k}$ for $i \in I \backslash\{m\}$, the above term gets as negative as we wish. Now, if we let $x_{m}$ tend to zero, then we obtain a contradiction. Next, $[i \mathrm{~km}]=0$ for $i \in I \backslash\{m\}$ and $k \in I^{C}$, since if we let tend $x_{k}$ to zero while keeping $x_{i}$ fixed the above term gets again as negative as we wish. Finally we obtain (4.1).

Let $w_{k}=\frac{x_{k}}{x_{m}}$ for $k \neq m$. By (4.1), equation (2.2) simplifies to

$$
\begin{aligned}
& \left(\left(\left(2 \operatorname{Ric}_{g}\right)_{I_{*}}\right)^{0}\right)_{m m} \cdot x_{m} \cdot d_{I} \\
& =\left(\frac{d_{I}}{d_{m}}-1\right)\left(d_{m} b_{m}+\frac{1}{2}[m m m]-\sum_{k}[k k m]\right) \\
& \quad-\sum_{i \in I \neq m} \frac{1}{w_{i}}\left\{d_{i} b_{i}+\frac{1}{2}[i i i]-\sum_{k}[i k k]\right\}+\frac{1}{2}\left(\frac{d_{I}}{d_{m}}+1\right) \sum_{i \in I \neq m}[i i m] \frac{1}{w_{i}^{2}} \\
& \quad+\frac{1}{2}\left(\frac{d_{I}}{d_{m}}-1\right) \sum_{k \in I^{C}}[k k m] \frac{1}{w_{k}^{2}}+\sum_{i \in I \neq k \neq m}[i i k] \frac{w_{k}}{w_{i}^{2}}-\frac{1}{2} \sum_{i \in I \neq k \neq m}[i k k] \frac{w_{i}}{w_{k}^{2}} \\
& \quad+\sum_{i \in I \neq j \neq k \neq m}[i j k] \frac{w_{k}}{w_{i} w_{j}}-\frac{1}{2} \sum_{i \in I \neq j \neq k \neq m}[i j k] \frac{w_{i}}{w_{j} w_{k}} .
\end{aligned}
$$

For all $j, k \in I^{C}$ we set $w_{j}=w_{k}$. Let $w_{i}$ be fixed for all $i \in I \backslash\{m\}$ but large enough. Now let $w_{k}$ tend to zero. We get

$$
[i j k]=0 \quad \text { for } i \in I \backslash\{m\}, j, k \in I^{C} .
$$

Hence, by (4.1) and (4.3) $\mathfrak{l}=\mathfrak{h} \oplus \mathfrak{m}_{m} \oplus \bigoplus_{k \in I^{C}} \mathfrak{m}_{k}$ is an $H$-subalgebra.

By (4.3) equation (4.2) simplifies to

$$
\begin{aligned}
& \left(\left(\left(4 \mathrm{Ric}_{g}\right)_{I_{*}}\right)^{0}\right)_{m m} \cdot x_{m} \cdot d_{I}=2\left(\frac{d_{I}}{d_{m}}-1\right)\left(d_{m} b_{m}+\frac{1}{2}[\mathrm{mmm}]-\sum_{i}[i i m]\right) \\
& -2 \sum_{i \in I \neq m} \frac{1}{w_{i}}\left\{d_{i} b_{i}+\frac{1}{2}[i i i]-\sum_{k \in I}[i k k]\right\}+\left(\frac{d_{I}}{d_{m}}+1\right) \sum_{i \in I \neq m}[i i m] \frac{1}{w_{i}^{2}} \\
& +\sum_{i \in I \neq j \in I \neq m}[i i j] \frac{w_{j}}{w_{i}^{2}}+\sum_{i \in I \neq j \in I \neq k \in I \neq m}[i j k] \frac{w_{k}}{w_{i} w_{j}} \\
& +\left(\frac{d_{I}}{d_{m}}-1\right) \sum_{k \in I^{C}}[k k m] \frac{1}{w_{k}^{2}}+2 \sum_{i \in I \neq m, k \in I^{C}}[i i k] \frac{w_{k}}{w_{i}^{2}} \\
& +2 \sum_{i \in I \neq j \in I \neq m, k \in I^{C}}[i j k] \frac{w_{k}}{w_{i} w_{j}}
\end{aligned}
$$

and we obtain the following counterpart to Proposition 3.7. 
Proposition 4.4. Let $G / H$ be a compact homogeneous space. Let $I_{*} \subset\left\{1,2, \ldots, \ell_{*}\right\}$ and $i_{0} \in I_{*}$. Suppose that $\left(\left(\left(\operatorname{Ric}_{g}\right)_{I_{*}}\right)^{0}\right)_{i_{0}}$ is positive definite for all $G$-invariant metrics $g$ on $G / H$. If $\mathfrak{p}_{i_{0}}$ is not irreducible, then virtually $G / H=T^{k} \times G_{2} / H_{2}$ for $k \geq 2$. Furthermore, if $\mathfrak{p}_{i_{0}}$ is irreducible and $\mathfrak{h} \oplus \mathfrak{p}_{i_{0}}$ is a toral H-subalgebra, the same is true for $k \geq 1$.

Proof. If $\mathfrak{p}_{i_{0}}$ is not irreducible, then we conclude as above that $\mathfrak{p}_{i_{0}}$ is an abelian subalgebra of $\mathfrak{g}$. Consequently, we obtain from (1.4) and (4.1) $d_{m} b_{m}+\frac{1}{2}[\mathrm{mmm}]-$ $\sum_{i}[i i m]=2 d_{m} c_{m}+\frac{1}{2}[\mathrm{mmm}]+\sum_{i \neq j}[i j m]=0$. Next, we set $w_{i}=x$ for $i \in I \backslash\{m\}$ and $w_{k}=x^{\frac{2}{3}}$ for $k \in I^{C}$ and let $x$ tend to $+\infty$. Since by assumption $\left(\left(\left(2 \mathrm{Ric}_{g}\right)_{I_{*}}\right)^{0}\right)_{m m}$ is positive and since by (4.3) $d_{i} b_{i}+\frac{1}{2}[i i i]-\sum_{k \in I}[i k k]=2 d_{i} c_{i}+$ $\frac{1}{2}[i i i]+\sum_{j \neq k}[i j k]$ for all $i \in I \backslash\{m\}$ we conclude $c_{i}=[i i i]=\sum_{j \neq k}[i j k]=0$ for $i \in I \backslash\{m\}$. It follows that $\bigoplus_{i \in I \neq m} \mathfrak{m}_{i}$ is an abelian subalgebra of $\mathfrak{g}$ which commutes with $\mathfrak{l}=\mathfrak{h} \oplus \mathfrak{m}_{m} \oplus \bigoplus_{k \in I^{C}} \mathfrak{m}_{k}$.

In the remaining part of this section we will assume $\left|\pi_{1}(G / H)\right|<\infty$. In this case it follows from the above proposition that $\mathfrak{p}_{i_{0}}$ is irreducible and that $\mathfrak{h} \oplus \mathfrak{p}_{i_{0}}$ is not a toral subalgebra of $\mathfrak{g}$. Therefore, the Lie subgroup $K=H\left(\mathfrak{h} \oplus \mathfrak{p}_{i_{0}}\right)$ is compact with $\operatorname{dim} K / H>1$. By (4.1) the $\operatorname{Ad}(H)$-irreducible summands $\mathfrak{m}_{i}, i \neq m$, are also $\operatorname{Ad}(K)$-invariant. Thus, all $G$-invariant metrics on $G / H$ are Riemannian submersion metrics with respect to the submersion $\pi: G / H \rightarrow G / K ; g K \mapsto g H$ with fibre $K / H$. We obtain the proof of Theorem $\mathrm{C}$.

Let us turn to compact homogeneous spaces where not only (4.3) is fulfilled but

$$
[i j k]=0 \quad \text { for } i \in I, j, k \in I^{C} .
$$

Then both $\mathfrak{h} \oplus \bigoplus_{k \in I^{C}} \mathfrak{m}_{k}$ and $\mathfrak{h} \oplus \mathfrak{m}_{m}$ are subalgebras of $\mathfrak{g}$. Under this assumption, we do not only ask $\left(\left(\left(\operatorname{Ric}_{g}\right)_{I_{*}}\right)^{0}\right)_{i_{0}}$ to be positive definite for all $g \in \mathcal{M}^{G}$ but require in addition that the following inequality is fulfilled:

$$
\begin{aligned}
0<2( & \left.\frac{d_{I}}{d_{m}}-1\right)\left(d_{m} b_{m}+\frac{1}{2}[\mathrm{mmm}]-\sum_{i \in I}[i i m]\right) \\
& -\sum_{i \in I \neq m} \frac{2}{w_{i}}\left\{d_{i} b_{i}+\frac{1}{2}[i i i]-\sum_{k \in I}[i k k]\right\}+\left(\frac{d_{I}}{d_{m}}+1\right) \sum_{i \in I \neq m}[i i m] \frac{1}{w_{i}^{2}} \\
& +\sum_{i \in I \neq j \in I \neq m}[i i j] \frac{w_{j}}{w_{i}^{2}}+\sum_{i \in I \neq j \in I \neq k \in I \neq m}[i j k] \frac{w_{k}}{w_{i} w_{j}} .
\end{aligned}
$$

Notice that this inequality is only a slightly stronger assumption than requiring $\left(\left(\left(\operatorname{Ric}_{g}\right)_{I_{*}}\right)^{0}\right)_{i_{0}}$ to be positive definite.

Now we can state the glueing result for homogeneous spaces mentioned in the introduction. 
Theorem 4.7. Let $G / H, \tilde{G} / \tilde{H}$ be compact homogeneous spaces with finite fundamental group. Suppose that there exists a simple Lie algebra $\mathfrak{l}$ such that $T_{1} H=\mathfrak{h}=\mathfrak{h}^{\prime} \oplus \mathfrak{l}$ and $T_{1} \tilde{H}=\tilde{\mathfrak{h}}=\mathfrak{l} \oplus \tilde{\mathfrak{h}}^{\prime}$. Let $\hat{G}=G \times \tilde{G}$ and let $\hat{H}$ denote a (possibly disconnected) subgroup of $H \times \tilde{H}$ with Lie algebra $\hat{\mathfrak{h}}=\mathfrak{h}^{\prime} \oplus \Delta \mathfrak{l} \oplus \tilde{\mathfrak{h}}^{\prime}$. Let $\mathfrak{m}=\bigoplus_{i=1}^{\ell_{*}} \mathfrak{p}_{i}$ denote the isotypical decomposition of the isotropy representation $\mathfrak{m}$ of $H$. Let $I_{*} \subset\left\{1,2, \ldots, \ell_{*}\right\}$ and $i_{0} \in I_{*}$. If

(1) $\left(\left(\left(\operatorname{Ric}_{g}\right)_{I_{*}}\right)^{0}\right)_{i_{0}}$ is positive definite and (4.6) is fulfilled for all $g \in \mathcal{M}^{G}$,

(2) for $i \in I_{*}$ the $\operatorname{Ad}(H)$-isotypical summands $\mathfrak{p}_{i}$ are $\operatorname{Ad}(\hat{H})$-isotypical summands of $\hat{\mathfrak{m}}$,

(3) the $\operatorname{Ad}(H)$-irreducible summands of $\bigoplus_{i \in I_{*}} \mathfrak{p}_{i}$ are $\operatorname{Ad}(\hat{H})$-irreducible,

(4) $\mathfrak{h} \oplus \bigoplus_{i \in\left\{1, \ldots, \ell_{*}\right\} \backslash I_{*}} \mathfrak{p}_{i}$ is a subalgebra of $\mathfrak{g}$ and $\left[\mathfrak{p}_{i_{0}}, \mathfrak{l}\right]=0$,

then $\hat{G} / \hat{H}$ does not admit $\hat{G}$-invariant Einstein metrics.

Proof. For the same choice of $I_{*}$ and $i_{0} \subset I_{*}$ we have to prove that (4.1), (4.5) and (4.6) are fulfilled for all $\hat{G}$-invariant metrics $g$ on $\hat{G} / \hat{H}$.

The isotropy representation $\hat{\mathfrak{m}}$ of $\hat{H}$ can be decomposed as follows:

$$
\hat{\mathfrak{m}}=\tilde{\mathfrak{m}} \oplus(\mathfrak{l} \oplus \mathfrak{l} \ominus \Delta \mathfrak{l}) \oplus\left(\bigoplus_{i \in I_{*}^{C}} \mathfrak{p}_{i}\right) \oplus\left(\bigoplus_{i \in I_{*}} \mathfrak{p}_{i}\right),
$$

where $\tilde{\mathfrak{m}}$ denotes the isotropy representation of $\tilde{H}$ and $I_{*}^{C}=\left\{1,2, \ldots, \ell_{*}\right\} \backslash I_{*}$. By (2), for $i \in I_{*}$ the summands $\mathfrak{p}_{i}$ are still isotypical summands of $\hat{\mathfrak{m}}$.

Let $\hat{f}=\bigoplus_{i \in I^{\hat{C}}} \hat{\mathfrak{m}}_{i} \oplus \bigoplus_{i \in I} \mathfrak{m}_{i}$ be an arbitrary decomposition of $\hat{\mathfrak{m}}$ into $\operatorname{Ad}(\hat{H})$ irreducible summands, where $I^{\hat{C}}=\{1,2, \ldots, \hat{\ell}\} \backslash I$ and $\bigoplus_{i \in I} \mathfrak{m}_{i}=\bigoplus_{i \in I_{*}} \mathfrak{p}_{i}, \mathfrak{m}_{i}$ $\operatorname{Ad}(H)$-irreducible for $i \in I$ (cf. (3)). As above let $\mathfrak{m}_{m} \in \mathfrak{p}_{i_{0}}$.

First, we show $[j k m]_{\hat{f}}=0$ for $j \neq k$. For $j, k \in I$ this is certainly true by (4.1). In order to treat the other cases notice that $[\tilde{\mathfrak{m}}, \tilde{\mathfrak{m}}] \subset \tilde{\mathfrak{g}},[\mathfrak{l} \oplus \mathfrak{l} \ominus \Delta \mathfrak{l}, \mathfrak{l} \oplus \mathfrak{l} \ominus \Delta \mathfrak{l}] \subset \hat{\mathfrak{h}}$, $\left[\bigoplus_{i \in I_{*}^{C}} \mathfrak{p}_{i}, \bigoplus_{i \in I_{*}^{C}} \mathfrak{p}_{i}\right] \subset \mathfrak{h} \oplus \bigoplus_{i \in I_{*}^{C}} \mathfrak{p}_{i}$ by $(4),\left[\tilde{\mathfrak{m}}, \bigoplus_{i \in I_{*}^{C}} \mathfrak{p}_{i}\right]=0,[\mathfrak{l} \oplus \mathfrak{l} \ominus \Delta \mathfrak{l}, \tilde{\mathfrak{m}}] \subset \tilde{\mathfrak{m}}$ and $\left[\mathfrak{l} \oplus \mathfrak{l} \ominus \Delta \mathfrak{l}, \bigoplus_{i \in I_{*}^{C}} \mathfrak{p}_{i}\right] \subset \bigoplus_{i \in I_{*}^{C}} \mathfrak{p}_{i}$. Hence $[j k m]_{\hat{f}}=0$ for $j, k \notin I$. Finally, if $j \notin I$ but $k \in I$ we obtain again $[j k m]_{\hat{f}}=0$, since $\left[\bigoplus_{i \in I_{*}^{C}} \mathfrak{p}_{i}, \mathfrak{m}_{m}\right]=0$, $\left[\tilde{\mathfrak{m}}, \mathfrak{m}_{m}\right]=0$ and $\left[\mathfrak{l} \oplus \mathfrak{l} \ominus \Delta \mathfrak{l}, \mathfrak{m}_{m}\right]=0$ by (4).

As a further consequence we obtain $[i j k]=0$ for $i \in I$ and $j, k \in I^{\hat{C}}$. Since by (2) and (3) the structure constants $[i j k]_{\hat{f}}$ with $i, j, k \in I$ did not change we conclude that (4.6) is still satisfied.

Remark 4.8. The above theorem can also be proved for abelian subalgebras $\mathfrak{l}$ : In this case we require $\mathfrak{h}=\mathfrak{h}^{\prime} \oplus \mathfrak{a}^{\prime}$ and $\tilde{\mathfrak{h}}=\tilde{\mathfrak{a}}^{\prime} \oplus \tilde{\mathfrak{h}}^{\prime}$ where $\mathfrak{a}^{\prime}$ and $\tilde{\mathfrak{a}}^{\prime}$ denote the centers of $\mathfrak{h}$ and $\mathfrak{h}^{\prime}$, respectively. Then we consider compact Lie subalgebras $\hat{\mathfrak{h}}=\mathfrak{h}^{\prime} \oplus \Delta \mathfrak{a} \oplus \tilde{\mathfrak{h}}^{\prime}$ of $\mathfrak{h} \oplus \mathfrak{h}^{\prime}$, where now $\Delta \mathfrak{a}$ denotes any compact subalgebra of $\mathfrak{a}^{\prime} \oplus \tilde{\mathfrak{a}}^{\prime}$. 


\section{New non-existence examples}

In this section we describe many new compact homogeneous spaces $G / H$ with finite fundamental group, which do not admit $G$-invariant Einstein metrics. Certain combinations of the Einstein equations are considered, which can be written as a sum of squares in an obvious manner. This yields the desired non-existence examples.

Let $G / H$ be a compact homogeneous space with finite fundamental group. If the isotropy representation $\mathfrak{m}$ of the isotropy group $H$ is irreducible, then by Schur's Lemma, up to scaling, there exists only one symmetric $G$-invariant bilinear form on $G / H$. Consequently, each $G$-invariant metric is Einstein.

If the isotropy representation $\mathfrak{m}$ can be decomposed into two irreducible inequivalent summands $\mathfrak{m}_{1}$ and $\mathfrak{m}_{2}$, then the Einstein equation is given as follows:

$$
\begin{aligned}
& \frac{1}{x_{1}}\left(\frac{b_{1}}{2}-\frac{[111]}{4 d_{1}}-\frac{[122]}{2 d_{1}}\right)-\frac{[112]}{2 d_{1}} \cdot \frac{x_{2}}{x_{1}^{2}}+\frac{[122]}{4 d_{1}} \cdot \frac{x_{1}}{x_{2}^{2}}=\lambda \\
& \frac{1}{x_{2}}\left(\frac{b_{2}}{2}-\frac{[222]}{4 d_{2}}-\frac{[112]}{2 d_{2}}\right)-\frac{[122]}{2 d_{2}} \cdot \frac{x_{1}}{x_{2}^{2}}+\frac{[112]}{4 d_{2}} \cdot \frac{x_{2}}{x_{1}^{2}}=\lambda .
\end{aligned}
$$

For the definition of the (non-negative) structure constants $[i j k]$ and $b_{1}, b_{2}$ we refer to Section 2. Recall that $[i j k]$ is invariant under permutation of $i, j, k$.

If [112], [221] $>0$, then $\mathfrak{h}$ is a maximal subalgebra of $\mathfrak{g}$, hence by [WZ2] there exists a positive real solution. Therefore, we may assume that $\mathfrak{h} \oplus \mathfrak{m}_{1}$ is the Lie algebra of an intermediate Lie group $\mathrm{K}$, that is [112] $=0$. If [122] $=0$ as well, then there exists a positive real solution since $\left|\pi_{1}(G / H)\right|<\infty$. Hence we may assume that [112] $=0$ and [122] $>0$. As was already proved in [WZ2], in this case the above system does not admit real solutions if and only if

$$
\left(b_{1}-\frac{[111]}{2 d_{1}}-\frac{[122]}{d_{1}}\right) \cdot[122] \cdot\left(\frac{1}{2 d_{1}}+\frac{1}{d_{2}}\right)>\frac{1}{4} \cdot\left(b_{2}-\frac{[222]}{2 d_{2}}\right)^{2} .
$$

If $G$ is simple and $G / K$ and $K / H$ are symmetric spaces, then $b=b_{1}=b_{2}$ and $[111]=[222]=0$, hence non-existence is guaranteed if and only if

$$
\left(b-\frac{[122]}{d_{1}}\right) \cdot[122] \cdot\left(\frac{1}{2 d_{1}}+\frac{1}{d_{2}}\right)>\frac{b^{2}}{4} .
$$

In [WZ2] many examples $G / H$ of this kind have been described with $G$ simple. This work has been completed in the recent classification of all theses spaces [DiKe]. For instance the homogeneous space $G / H=\mathrm{SU}(m+n) / \mathrm{S}(\mathrm{SO}(m) \mathrm{U}(1) \mathrm{U}(n))$ does not admit $G$-invariant Einstein metrics for $m>n^{2}+2$. If $m=n^{2}+2$, then $G / H$ admits precisely one $G$-invariant Einstein metric, whereas for $m<n^{2}+2$ there are two non-isometric $G$-invariant Einstein metrics. 
In order to describe further non-existence examples, we consider compact irreducible symmetric spaces $\tilde{G} /\left(\tilde{H}_{1} \tilde{H}_{2}\right)$, such that $\tilde{H}_{1}$ is simple and $\tilde{H}_{2}$ is either simple or 1-dimensional (cf. [Bes, Table 7.102]). We examine the homogeneous spaces

$$
G / H=\tilde{G} \times \tilde{G} /\left(\Delta \tilde{H}_{1} \cdot\left(\tilde{H}_{2} \times \tilde{H}_{2}\right)\right)
$$

where the subgroup $\Delta \tilde{H}_{1}$ denotes the diagonal embedding of $\tilde{H}_{1}$ in $\tilde{H}_{1} \times \tilde{H}_{1}$.

The isotropy representation $\mathfrak{m}$ of $H$ consists of three pairwise inequivalent summands given by $\mathfrak{m}_{1}=\tilde{\mathfrak{g}}_{1} \ominus\left(\tilde{\mathfrak{h}}_{1} \oplus \tilde{\mathfrak{h}}_{2}\right)_{1}, \mathfrak{m}_{2}=\tilde{\mathfrak{g}}_{2} \ominus\left(\tilde{\mathfrak{h}}_{1} \oplus \tilde{\mathfrak{h}}_{2}\right)_{2}$ and $\mathfrak{m}_{3}=\left(\tilde{\mathfrak{h}}_{1} \oplus \tilde{\mathfrak{h}}_{1}\right) \ominus \Delta \tilde{\mathfrak{h}}_{1}$. We have $d_{1}=d_{2}=\operatorname{dim} \mathfrak{m}_{1}=\operatorname{dim} \tilde{G}-\operatorname{dim} \tilde{H}_{1}-\operatorname{dim} \tilde{H}_{2}$ and $d_{3}=\operatorname{dim} \mathfrak{m}_{3}=\operatorname{dim} \tilde{H}$. It is easy to see that the only non-vanishing structure constants are [113] and [223]. By choosing an $\operatorname{Ad}(G)$-invariant scalar product on $\mathfrak{g}$ whose restriction to both simple factors agrees, we get $b=b_{1}=b_{2}=b_{3}>0$ and [113] $=$ [223]. A routine computation using (1.3) shows that the Einstein equation is given as follows:

$$
\begin{array}{r}
\frac{b}{2 x_{1}}-\frac{[113]}{2 d_{1}} \cdot \frac{x_{3}}{x_{1}^{2}}=\lambda \\
\frac{b}{2 x_{2}}-\frac{[113]}{2 d_{1}} \cdot \frac{x_{3}}{x_{2}^{2}}=\lambda \\
\frac{1}{x_{3}}\left(\frac{b}{2}-\frac{[113]}{d_{3}}\right)+\frac{[113]}{4 d_{3}} \cdot \frac{x_{3}}{x_{1}^{2}}+\frac{[113]}{4 d_{3}} \cdot \frac{x_{3}}{x_{2}^{2}}=\lambda .
\end{array}
$$

In order to examine the non-existence criterion described in Theorem $\mathrm{C}$ let us compute the restriction of the tracefree part of the Ricci tensor restricted to $\mathfrak{m}_{3}$ (that is we choose $I_{*}=\{1,2,3\}$ and $\left.i_{0}=3\right)$. Up to a factor we consider the equation $x_{3} \cdot(2(5.4)-(5.2)-(5.3))=0$ given by

$$
\frac{[113]}{2} \cdot\left(\frac{1}{d_{1}}+\frac{1}{d_{3}}\right) \cdot\left(\alpha^{2}+\beta^{2}\right)-\frac{b}{2} \cdot(\alpha+\beta)+\left(b-\frac{2[113]}{d_{3}}\right)=0
$$

where $\alpha=\frac{x_{3}}{x_{1}}$ and $\beta=\frac{x_{3}}{x_{2}}$. It follows that if

$$
\left(b-\frac{2[113]}{d_{3}}\right) \cdot[113] \cdot\left(\frac{1}{d_{1}}+\frac{1}{d_{3}}\right)>\frac{b^{2}}{4},
$$

then the system (5.2), (5.3), (5.4) does not admit real solutions.

Example 5.7. The spaces $G / H=\mathrm{SO}(n) \times \mathrm{SO}(n) / \Delta \mathrm{SO}(n-k) \cdot(\mathrm{SO}(k) \times \mathrm{SO}(k))$ do not admit $G$-invariant Einstein metrics for $n>k^{2}+k+2$ and $k \geq 2$.

Proof. We choose the $\operatorname{Ad}(G)$-invariant scalar product $Q(X, Y)=-\frac{1}{2} \operatorname{tr}(X \cdot Y)$ on $\mathfrak{g}$. Then $b=2(n-2)$ (see [WZ1, p. 583]). Furthermore $d_{1}=d_{2}=k(n-k)$ and $d_{3}=\frac{1}{2}(n-k)(n-k-1)$. A computation shows [113] $=k d_{3}$ and we obtain the claim from (5.6). 
It is not hard to see, that $G / H$ admits $G$-invariant Einstein metrics if the obstruction (5.6) is violated; for $n=k^{2}+k+2$ there exists a unique $G$-invariant Einstein metric and for $n<k^{2}+k+2$ there exist at least two non-isometric Einstein metrics.

In the next step we specialize to symmetric spaces $\tilde{G} /(\tilde{H} \mathrm{SO}(2))$, where $\tilde{H}$ is a simple Lie group (cf. [Bes, Table 7.102]). For coprime integers $p, q$ with $(p, q) \neq$ $\pm(1,1)$, we consider the homogeneous spaces

$$
G / H=\tilde{G} \times \tilde{G} /\left(\Delta \tilde{H} \cdot \mathrm{SO}_{p, q}(2)\right)
$$

where $\mathrm{SO}_{p, q}(2)$ is embedded diagonally in $\mathrm{SO}(2) \times \mathrm{SO}(2)$ with slope determined by $(p, q)$.

Since $p \neq q$ the isotropy representation $\mathfrak{m}$ consists of four pairwise inequivalent summands given by $\mathfrak{m}_{1}=\tilde{\mathfrak{g}}_{1} \ominus\left(\mathfrak{s o}(2)_{1} \oplus \tilde{\mathfrak{h}}_{1}\right), \mathfrak{m}_{2}=\tilde{\mathfrak{g}}_{2} \ominus\left(\mathfrak{s o}(2)_{2} \oplus \tilde{\mathfrak{h}}_{2}\right), \mathfrak{m}_{3}=$ $(\tilde{\mathfrak{h}} \oplus \tilde{\mathfrak{h}}) \ominus \Delta \tilde{\mathfrak{h}}$ and $\mathfrak{m}_{4}=\left(\mathfrak{s o}(2)_{1} \oplus \mathfrak{s o}(2)_{2}\right) \ominus \mathfrak{s o} \mathfrak{s}_{p, q}(2)$. We have $d_{1}=d_{2}=\operatorname{dim} \mathfrak{m}_{1}=$ $\operatorname{dim} \tilde{G}-\operatorname{dim} \tilde{H}-1, d_{3}=\operatorname{dim} \mathfrak{m}_{3}=\operatorname{dim} \tilde{H}$ and $d_{4}=\operatorname{dim} \mathfrak{m}_{4}=1$. It is easy to see that the only non-vanishing structure constants are [113], [114], [223] and [224]. By choosing an $\operatorname{Ad}(G)$-invariant scalar product on $\mathfrak{g}$ whose restriction to both simple factors agrees, we get $b=b_{1}=b_{2}=b_{3}=b_{4}>0$ and [113] $=$ [223]. Since the Casimir constant $c_{4}$ of the irreducible summand $\mathfrak{m}_{4}$ equals zero, by (1.4) we obtain

$$
b=b_{4}=[411]+[422] .
$$

A computation shows that the Einstein equation is given as follows:

$$
\begin{array}{r}
\frac{b}{2 x_{1}}-\frac{[113]}{2 d_{1}} \cdot \frac{x_{3}}{x_{1}^{2}}-\frac{[114]}{2 d_{1}} \cdot \frac{x_{4}}{x_{1}^{2}}=\lambda \\
\frac{b}{2 x_{2}}-\frac{[113]}{2 d_{1}} \cdot \frac{x_{3}}{x_{2}^{2}}-\frac{[224]}{2 d_{1}} \cdot \frac{x_{4}}{x_{2}^{2}}=\lambda \\
\frac{1}{x_{3}}\left(\frac{b}{2}-\frac{[113]}{d_{3}}\right)+\frac{[113]}{4 d_{3}} \cdot \frac{x_{3}}{x_{1}^{2}}+\frac{[113]}{4 d_{3}} \cdot \frac{x_{3}}{x_{2}^{2}}=\lambda \\
\frac{x_{4}}{4 d_{4}}\left([114] \cdot \frac{1}{x_{1}^{2}}+[224] \cdot \frac{1}{x_{2}^{2}}\right)=\lambda .
\end{array}
$$

We consider the equation $-4 x_{3}\left(\frac{d_{1}}{2}(5.8)+\frac{d_{1}}{2}(5.9)+d_{4}(5.11)-\left(d_{1}+d_{4}\right)(5.10)\right)=0$ given by

$\frac{[113]}{d_{3}} \cdot\left(d_{1}+d_{3}+d_{4}\right) \cdot\left(\alpha^{2}+\beta^{2}\right)-d_{1} b \cdot(\alpha+\beta)+\left(d_{1}+d_{4}\right) \cdot\left(2 b-\frac{4[113]}{d_{3}}\right)=0$, where again $\alpha=\frac{x_{3}}{x_{1}}$ and $\beta=\frac{x_{3}}{x_{2}}$. It follows that if

$$
\left(b-\frac{2[113]}{d_{3}}\right) \cdot[113] \cdot \frac{\left(d_{1}+d_{3}+d_{4}\right)\left(d_{1}+d_{4}\right)}{d_{1}^{2} d_{3}}>\frac{b^{2}}{4},
$$


then the system (5.8), (5.9), (5.10), (5.11) does not admit real solutions.

This non-existence criterion is obtained by combining two non-existence criteria described in Lemma 1.2. In the above equation we consider a weighted sum of $\left(\left(\operatorname{Ric}_{\{1,2,3\}}\right)^{0}\right)_{3}$ and $\left(\left(\operatorname{Ric}_{\{3,4\}}\right)^{0}\right)_{3}$.

Example 5.13 ([BK]). Let $n \geq 3$ and let $p, q$ be coprime integers with $(p, q) \neq$ $\pm(1,1)$. Then the space $G / H=\mathrm{SU}(n) \times \mathrm{SU}(n) /\left(\Delta \mathrm{SU}(n-1) \cdot \mathrm{U}_{p, q}(1)\right)$ does not admit $G$-invariant Einstein metrics.

Proof. The embedding of $H$ into $G$ is given as follows: Consider the maximal subgroup $\mathrm{U}(n-1)$ in $\mathrm{SU}(n)$. Then the semisimple part of $H$ is embedded diagonally and $\mathrm{U}_{p, q}(1)$ is embedded into the center of $\mathrm{U}(n-1) \times \mathrm{U}(n-1)$ with slope determined by $(p, q)$.

We choose the $\operatorname{Ad}(G)$-invariant scalar product $Q(X, Y)=-\frac{1}{2} \operatorname{tr}(X \cdot Y)$ on $\mathfrak{g}$. Then $b=4 n$ (see [WZ1, p. 583]). Furthermore $d_{1}=d_{2}=2(n-1), d_{3}=n(n-2)$ and $d_{4}=1$. A computation shows [113] $=2 d_{3}$ and the claim follows from (5.12).

For $n=3$ this example has been examined in [BK] as one of the 12-dimensional homogeneous spaces which do not admit homogeneous Einstein metrics. It is interesting to note that for $(p, q)= \pm(1,1) G / H$ carries a $G$-invariant Einstein metric by the Graph Theorem [BWZ]. In this case the irreducible summands $\mathfrak{m}_{1}$ and $\mathfrak{m}_{2}$ are equivalent and therefore, the space of $G$-invariant metrics is 6-dimensional. Since the above non-existence proof does not rely on the particular values of $(p, q)$, we conclude that this Einstein metric is not contained in the 4-dimensional family of $G$-invariant metrics described above.

Next, we describe a second non-existence criterion for real solutions of the system (5.8), (5.9), (5.10), (5.11). As in (5.5) we consider the equation $x_{3} \cdot(2(5.10)-(5.8)-$ $(5.9))=0$, which up to a factor is nothing but $\left(\left(\operatorname{Ric}_{\{1,2,3\}}\right)^{0}\right)_{3}$. We obtain

$$
\begin{aligned}
& \frac{[113]}{2} \cdot\left(\frac{1}{d_{1}}+\frac{1}{d_{3}}\right) \cdot\left(\alpha^{2}+\beta^{2}\right)-\frac{b}{2} \cdot(\alpha+\beta) \\
& \quad+\left(b-\frac{2[113]}{d_{3}}\right)+\frac{[114]}{2 d_{1}} \cdot \frac{x_{3} x_{4}}{x_{1}^{2}}+\frac{[224]}{2 d_{1}} \cdot \frac{x_{3} x_{4}}{x_{2}^{2}}=0,
\end{aligned}
$$

where as above $\alpha=\frac{x_{3}}{x_{1}}$ and $\beta=\frac{x_{3}}{x_{2}}$. Since [114], [224] $\geq 0$, [114] + [224] $>0$ and $x_{1}, \ldots, x_{4}>0$, for the homogeneous space $\tilde{G} \times \tilde{G} /(\Delta \tilde{H} \cdot(\operatorname{SO}(2) \times \operatorname{SO}(2)))$ the equations (5.8), (5.9), (5.10), (5.11) do not have real solutions, if the non-existence criterion (5.6) is satisfied. Notice that this criterion in weaker than that described in (5.12); for instance non-existence for Example 5.13 does not follow from (5.6).

Example 5.14. Let $n \geq 7$ and let $p, q$ be coprime integers with $(p, q) \neq(1,1)$. Then the compact homogeneous space $G / H=\mathrm{SO}(n) \times \mathrm{SO}(n) /\left(\Delta \mathrm{SO}(n-2) \cdot \mathrm{SO}_{p, q}(2)\right)$ does not admit $G$-invariant Einstein metrics. 
Proof. The embedding of $H$ into $G$ is as described in Example 5.13. As in Example 5.7 we choose the $\operatorname{Ad}(G)$-invariant scalar product $Q(X, Y)=-\frac{1}{2} \operatorname{tr}(X \cdot Y)$ on $\mathfrak{g}$, hence $b=2(n-2)$. Furthermore $d_{1}=d_{2}=2(n-2), d_{3}=\frac{1}{2}(n-2)(n-3)$ and $d_{4}=1$. In the proof of Example 5.7 we saw [113] $=[223]=2 d_{3}$. Non-existence of $G$-invariant Einstein metrics follows now from (5.6) for $n>8$. For $n=8$ we have equality in (5.6), which still implies non-existence of $G$-invariant Einstein metrics on $G / H$. For $n=7$ we need to invoke (5.12) and the claim follows.

Next, let us give the proof of Theorem A. Let $G / H$ be a compact simply connected homogeneous space with a prime factor $G_{p} / H_{p}=\tilde{G} \times \tilde{G} /(\Delta \tilde{H} \cdot(\operatorname{SO}(2) \times \operatorname{SO}(2)))$. Then either

$$
G / H=G_{p} / H_{p} \times \hat{G} / \hat{T} \hat{H}_{s} \quad \text { or } \quad G / H=\tilde{G} \times \tilde{G} \times \hat{G} /\left(\Delta \tilde{H} \cdot \Delta T \cdot \hat{H}_{s}\right)
$$

where $\hat{G} / \hat{T} \hat{H}_{s}$ is a product of prime homogeneous spaces, $\hat{T}$ denotes the center of $\hat{T} \hat{H}_{S}$ (on Lie algebra level), and $\Delta T$ is a proper subtorus of $(\mathrm{SO}(2) \times \mathrm{SO}(2)) \times \hat{T}$.

In the first case $G / H$ does not admit $G$-invariant Einstein metrics, since the isotropy representation of $G_{p} / H_{p}$ does not contain trivial summands.

In the second case, under the genericity assumption the summands $\mathfrak{m}_{1}, \mathfrak{m}_{2}$ and $\mathfrak{m}_{3}$ of the isotropy representation of $H_{p}$ are still irreducible isotypical summands of the isotropy representation $\mathfrak{m}=\mathfrak{m}_{1} \oplus \mathfrak{m}_{2} \oplus \mathfrak{m}_{3} \oplus \bigoplus_{i=4}^{\ell} \mathfrak{m}_{i}$ of $H$. Notice that the decomposition $\bigoplus_{i=4}^{\ell} \mathfrak{m}_{i}$ of $\mathfrak{m} \ominus\left(\mathfrak{m}_{1} \oplus \mathfrak{m}_{2} \oplus \mathfrak{m}_{3}\right)$ may not be uniquely determined. Still the Einstein equations, which correspond to (5.8), (5.9) and (5.10), are given by

$$
\begin{array}{r}
\frac{b_{1}}{2 x_{1}}-\frac{[113]}{2 d_{1}} \cdot \frac{x_{3}}{x_{1}^{2}}-\sum_{i=4}^{\ell} \frac{[11 i]}{2 d_{1}} \cdot \frac{x_{i}}{x_{1}^{2}}=\lambda \\
\frac{b_{2}}{2 x_{2}}-\frac{[223]}{2 d_{1}} \cdot \frac{x_{3}}{x_{2}^{2}}-\sum_{i=4}^{\ell} \frac{[22 i]}{2 d_{1}} \cdot \frac{x_{i}}{x_{2}^{2}}=\lambda \\
\frac{1}{x_{3}}\left(\frac{b_{3}}{2}-\frac{[113]}{d_{3}}\right)+\frac{[113]}{4 d_{3}} \cdot \frac{x_{3}}{x_{1}^{2}}+\frac{[223]}{4 d_{3}} \cdot \frac{x_{3}}{x_{2}^{2}}=\lambda .
\end{array}
$$

By choosing an $\operatorname{Ad}(G)$-invariant scalar product on $\mathfrak{g}$ which extends that described in the proof of Example 5.14, we conclude $b=b_{1}=b_{2}=b_{3}$. Furthermore, we still have $[113]=[223]=2 d_{3}$. As above we conclude, that if the non-existence criterion (5.6) is fulfilled, then the Einstein equations for $G / H$ do not admit real solutions. This completes the proof of Theorem A.

Remark 5.18. Observe that the above non-existence criterion is nothing but asking $\left(\left(\left(\operatorname{Ric}_{g}\right)_{I_{*}}\right)^{0}\right)_{3}: \mathfrak{m}_{3} \rightarrow \mathfrak{m}_{3}$ to be positive for all $G$-invariant metrics $g$ on $G / H$ for $I_{*}=\{1,2,3\}$ and $i_{0}=3$. Notice that we also could have applied Theorem 4.7. 
Finally, we describe one more elaborate non-existence example $G / H$.

Example 5.19. Let $G / H=\mathrm{SU}\left(m+n_{1}+\cdots+n_{k}\right) / \mathrm{S}\left(\mathrm{SO}(m) \mathrm{U}(1) \times \mathrm{U}\left(n_{1}\right) \times\right.$ $\left.\cdots \times \mathrm{U}\left(n_{k}\right)\right)$, where $m, n_{1}, \ldots, n_{k} \geq 1$. If $m>\left(\sum_{i=1}^{k} n_{i}\right)^{2}+2$, then $G / H$ does not admit $G$-invariant Einstein metrics.

Proof. The isotropy representation $\mathfrak{m}$ of $H$ can be decomposed as follows:

$$
\mathfrak{m}=\bigoplus_{1 \leq i \leq k}\left[\rho_{m} \otimes \phi \otimes \mu_{n_{i}}^{*}\right]_{\mathbb{R}} \oplus\left(\mathrm{S}^{2} \rho_{m}-\mathrm{Id}\right) \oplus \bigoplus_{1 \leq i<j \leq k}\left[\mu_{n_{i}} \otimes \mu_{n_{j}}^{*}\right]_{\mathbb{R}}
$$

All $\ell=k+1+\frac{1}{2} k(k-1)$ summands of $\mathfrak{m}$ are irreducible and pairwise inequivalent, hence $\ell=\ell_{*}$. Let the first $k+1$ summands be denoted by $\mathfrak{m}_{1}, \ldots, \mathfrak{m}_{k}, \mathfrak{m}_{m}$, for $m=k+1$. We set $I_{*}=I=\{1, \ldots, k+1\}$ and $i_{0}=m$. Note that (4.1) and (4.5) are satisfied. It remains to show that (4.6) is fulfilled as well.

We have $d_{m}=\frac{1}{2}(m+2)(m-1)$ and $d_{i}=2 m n_{i}$ for $i=1, \ldots, k$. This time we choose $Q=-B$ that is $b_{i}=1$ for all $i$. After rescaling the Killing form of $G / H$ a computation shows $[\mathrm{iim}]=d_{m} n_{i} /\left(m+\sum_{i=1}^{k} n_{i}\right)$ for $i=1, \ldots, k$ (cf. [WZ2], Example 2). All the other structure constants $[i j k]$ with $i, j, k \in I$ vanish. Now another computation involving the first three term in (4.6) shows that (4.6) is fulfilled for $m>\left(\sum_{i=1}^{k} n_{i}\right)^{2}+2$.

\section{References}

[AlKi] D. V. Alekseevsky, B. N. Kimel'fel'd, Structure of homogeneous Riemann spaces with zero Ricci curvature. Funct. Anal. Appl. 9 (1975), 97-102. Zbl 0316.53041 MR 0402650

[Bes] A. L. Besse, Einstein Manifolds. Ergeb. Math. Grenzgeb. 10, Springer-Verlag, Berlin 1987. Zbl 0613.53001 MR 0867684

[Bo] S. Bochner, Curvature and Betti numbers. Ann. of Math. 49 (1948), 379-390. Zbl 0038.34401 MR 0025238

[Bö1] C. Böhm, Non-existence of cohomogeneity one Einstein metrics. Math. Ann. 314 (1999), 109-125. Zbl 0959.53020 MR 1689265

[Bö2] C. Böhm, Homogeneous Einstein metrics and simplicial complexes. J. Differential Geom. 67 (2004), 79-165.

[BK] C. Böhm, M. Kerr, Low-dimensional homogeneous Einstein manifolds. To appear in Trans. Amer. Math. Soc.

[BWZ] C. Böhm, M. Y. Wang, W. Ziller, A variational approach for compact homogeneous Einstein manifolds. Geom. Funct. Anal. 14 (2004), 681-733.

[BoGa] C. P. Boyer, K. Galicki, On Sasakian-Einstein geometry. Internat. J. Math. 11 (2000), 873-909. Zbl 1022.53038 MR 1792957 
[DiKe] W. Dickinson, M. Kerr, The geometry of compact homogeneous spaces with two isotropy summands. Preprint 2004.

[GrZi] K. Grove, W. Ziller, Cohomogeneity one manifolds with positive Ricci curvature. Invent. Math. 149 (2002), 619-646. Zbl 1038.53034 MR 1923478

[Heb] J. Heber, Noncompact homogeneous Einstein spaces. Invent. Math. 133 (1998), 279-352. Zbl 0906.53032 MR 1632782

[Hi] D. Hilbert, Die Grundlagen der Physik. Nachr. Akad. Wiss. Gött. (1915), 395-407. JFM 45.1111.01

[Jo] D. Joyce, Compact manifolds with special holonomy. Oxford Math. Monogr., Oxford University Press, Oxford 2000. Zbl 1027.53052 MR 1787733

[LeB] C. LeBrun, Ricci curvature, minimal volumes, and Seiberg-Witten theory. Invent. Math. 145 (2001), 279-316. Zbl 0999.53027 MR 1872548

[LW] C. LeBrun, M. Y. Wang (eds.), Surveys in Differential Geometry VI: Essays on Einstein Manifolds. International Press, Cambridge, MA, 1999. Zbl 0961.00021 MR 1798603

[On] A. L. Onishchik, Topology of Transitive Transformation Groups. Johann Ambrosius Barth Verlag GmbH, Leipzig 1994. Zbl 0796.57001 MR 1266842

[PaSa] J.-S. Park, Y. Sakane, Invariant Einstein metrics on certain homogeneous spaces. Tokyo J. Math. 20 (1997), 51-61. Zbl 0884.53039 MR 1451858

[Tia] G. Tian, Kähler-Einstein metrics with positive scalar curvature. Invent. Math. 130 (1997), 1-37. Zbl 0892.53027 MR 1471884

[Wa] M. Y. Wang, Einstein metrics and quaternionic Kähler manifolds. Math. Z. 210 (1992), 305-325. Zbl 0765.53037 MR 1166528

[WZ1] M. Y. Wang, W. Ziller, On normal homogeneous Einstein manifolds. Ann. Sci. École Norm. Sup. (4) 18 (1985), 563-633. Zbl 0598.53049 MR 0839687

[WZ2] M. Y. Wang, W. Ziller, Existence and non-existence of homogeneous Einstein metrics. Invent. Math. 84 (1986), 177-194. Zbl 0596.53040 MR 0830044

[WZ3] M. Y. Wang, W. Ziller, On isotropy irreducible Riemannian manifolds. Acta Math. 199 (1991), 223-261. Zbl 0732.53040 MR 1097024

[Wo] J. A. Wolf, The geometry and structure of isotropy irreducible homogeneous spaces. Acta. Math. 120 (1968), 59-148; correction: Acta. Math. 152 (1984), 141-142. Zbl 0157.52102 Zbl 0539.53037 MR 0223501 MR 0736216

[Yau] S. T. Yau, On the Ricci curvature of a compact Kähler manifold and the complex Monge-Ampère equation I. Comm. Pure Appl. Math. 31 (1978), 339-411. Zbl 0369.53059 MR 0480350

Received July 28, 2003

Christoph Böhm, Mathematisches Institut, Universität Münster, Einsteinstrasse 62, 48149 Münster, Germany

E-mail: cboehm@math.uni-muenster.de 